\title{
NON-TOPOLOGICAL CONDENSATES IN SELF-DUAL CHERN-SIMONS GAUGE THEORY
}

\author{
TAKASHI SUZUKI \\ Division of Mathematical Science, Graduate School of Engineering Science \\ Osaka University, Osaka 650-8531, Japan \\ E-mail: suzuki@sigmath.es.osaka-u.ac.jp \\ FUTOSHI TAKAHASHI \\ Mathematical Institute, Tohoku University, Sendai 980-8578, Japan
}

\begin{abstract}
This note is concerned with the recent paper "Non-topological $N$-vortex condensates for the self-dual Chern-Simons theory" by M. Nolasco. Modifying her arguments and statements, we show that the existence of "non-topological" multi-vortex condensates follows when the number of prescribed vortex points is greater than or equal to 2 .
\end{abstract}

1. Introduction. In recent years, charged vortex solutions in $(2+1)$ dimensional abelian Chern-Simons vortex theory have received much attention, because of their relation to many fields of physics such as high-critical temperature superconductivity, some condensed matter systems, charged anyon-model, and so on ([5], [11]). A mathematical proof of the existence of stationary vortex solutions, called the vortex condensate, has been given by [1], [3], [4], [7], [8], [10] on the periodic cell domain satisfying a suitable gauge-invariant periodicity, that is, the 't Hooft boundary condition.

Let $\Omega$ be the fundamental cell domain in $\mathbf{R}^{2}$ generated by linearly independent vectors $e_{1}=(a, 0)$ and $e_{2}=(0, b)$ :

$$
\Omega=\left\{x=\left(x_{1}, x_{2}\right) \in \mathbf{R}^{2} \mid-\frac{a}{2} \leq x_{1} \leq \frac{a}{2}, \quad-\frac{b}{2} \leq x_{2} \leq \frac{b}{2}\right\},
$$

and let $p_{1}, \ldots, p_{s} \in \Omega \backslash \partial \Omega$ be $s$ distinct vortex points with multiplicities $m_{1}, \ldots, m_{s} \in$ $\mathbf{N}$. Then, after the reduction process of Taubes, existence of Chern-Simons $N$-vortex condensates is reduced to finding a solution $u=u_{\kappa}$ to

$$
\left\{\begin{array}{l}
-\Delta u=\frac{4}{\kappa^{2}} e^{u}\left(1-e^{u}\right)-4 \pi \sum_{j=1}^{s} m_{j} \delta_{p_{j}} \text { in } \Omega, \\
u: \text { doubly periodic on } \partial \Omega,
\end{array}\right.
$$

2000 Mathematics Subject Classification: 35J60, 35Q99.

The paper is in final form and no version of it will be published elsewhere. 
where $\kappa>0$ is the Chern-Simons coupling parameter and $\sum_{j=1}^{s} m_{j}=N$. From the maximum principle it follows that $e^{u}<1$ on $\Omega$, while integrating (1.1) over $\Omega$ implies

$$
\int_{\Omega} e^{u}\left(1-e^{u}\right) d x=\pi N \kappa^{2}
$$

Hence we obtain $e^{u_{\kappa}}\left(1-e^{u_{\kappa}}\right) \rightarrow 0$ a.e. as $\kappa \rightarrow 0$ passing through a subsequence. Actually, we are interested in the following cases:

- "topological" $N$-vortex condensates: $e^{u_{\kappa}} \rightarrow 1$ locally uniformly on $\Omega \backslash\left\{p_{1}, \ldots, p_{s}\right\}$ as $\kappa \rightarrow 0$.

- "non-topological" $N$-vortex condensates: $e^{u_{\kappa}} \rightarrow 0$ locally uniformly on $\Omega \backslash\left\{p_{1}, \ldots, p_{s}\right\}$ as $\kappa \rightarrow 0$.

The existence of "topological" $N$-vortex condensates was solved affirmatively in [10]. On the other hand, only partial results have been known concerning "non-topological" $N$-vortex condensates ([10], [8], [4], [3]).

In a recent paper, M. Nolasco asserted that "non-topological" $N$-vortex condensate existed for any given vortex points in $\Omega$. Unfortunately, some estimates are not described in detail and there seem to be several gaps in the argument. In this note, first, we shorten and clarify her analytic arguments. Second, we point out that some modifications are needed in the statement of the above theorem. More precisely, if the number of vortex points $s$ is greater than or equal to 2, then the conclusion of the above theorem is proven; more precisely,

Theorem 1. Given $p_{j} \in \Omega \backslash \partial \Omega$ and $m_{j} \in \mathbf{N}(j=1, \ldots, s)$, if $s \geq 2$ we have $\bar{\kappa}>0$ such that if $\kappa \in(0, \bar{\kappa})$ there is a solution $u=u_{\kappa}$ to (1.1) satisfying the following:

(1) $e^{u_{\kappa}}<1$ on $\Omega$.

(2) $e^{u_{\kappa}} \rightarrow 0$ in $C_{l o c}^{q}\left(\Omega \backslash\left\{p_{1}, \ldots, p_{s}\right\}\right.$ for any $q \geq 0$ as $\kappa \rightarrow 0$.

(3) $\frac{4}{\kappa^{2}} e^{u_{\kappa}}\left(1-e^{u_{\kappa}}\right) \rightarrow 4 \pi \sum_{j=1}^{s} m_{j} \delta_{p_{j}}$ in the sense of measures on $\Omega$ as $\kappa \rightarrow 0$.

In spite of the above mentioned technical improvements, we reproduce some parts of [9] for completeness. The authors thank Professor Dongho Chae for informing them about the original paper [9].

2. Radially symmetric vortex. First, we extend the solution $u=u(x)$ to (1.1) for all $x \in \mathbf{R}^{2}$ by periodicity. That is, $\widetilde{u}(x)=u\left(x+n_{1} e_{1}+n_{2} e_{2}\right)$, which satisfies that

$$
\begin{cases}-\Delta \widetilde{u}=\frac{4}{\kappa^{2}} e^{\widetilde{u}}\left(1-e^{\widetilde{u}}\right)-4 \pi \sum_{n \in \mathbf{Z}^{2}} \sum_{j=1}^{s} m_{j} \delta_{p_{j}^{n}} & \text { in } \mathbf{R}^{2}, \\ \widetilde{u}\left(x+e_{i}\right)=\widetilde{u}(x) & \text { for } x \in \mathbf{R}^{2} \text { and } i=1,2,\end{cases}
$$

where $p_{j}^{n}=p_{j}+n_{1} e_{1}+n_{2} e_{2}$ with $\left(n_{1}, n_{2}\right) \in \mathbf{Z}^{2}$ denotes the periodic lattice of vortex points for $j=1, \ldots, s$. Next, we introduce the scaling parameters $\delta, \varepsilon$ in $0<\delta \ll \varepsilon$ as $\kappa=2 \varepsilon \delta$, and set $\widehat{u}(x)=\widetilde{u}(\delta x)$ and $\widehat{p}_{j}^{n}=\frac{1}{\delta} p_{j}^{n}$ for $j=1, \ldots, s$. Then, it follows that

$$
\left\{\begin{array}{l}
-\Delta \widehat{u}=\frac{1}{\varepsilon^{2}} e^{\hat{u}}\left(1-e^{\hat{u}}\right)-4 \pi \sum_{n \in \mathbf{Z}^{2}} \sum_{j=1}^{s} m_{j} \delta_{\hat{p}_{j}^{n}} \quad \text { in } \mathbf{R}^{2}, \\
\widehat{u}\left(x+\widehat{e}_{i}\right)=\widehat{u}(x) \quad \text { for } x \in \mathbf{R}^{2} \text { and } i=1,2
\end{array}\right.
$$


for $\widehat{e}_{i}:=\frac{1}{\delta} e_{i}$, because we have $a^{2} \delta(x)=\delta\left(\frac{x}{a}\right)$ for any $a>0$ and $x \in \mathbf{R}^{2}$. This is the equation that we solve by the implicit function theorem, taking suitable approximate solutions. It is done by "glueing" radially symmetric vortex solutions of Chae-Imanuvilov [2], and therefore, we briefly recall the latter work.

Namely, we consider $N$-vortex condensate at the origin,

$$
\begin{cases}-\Delta u=\frac{1}{\varepsilon^{2}} e^{u}\left(1-e^{u}\right)-4 \pi N \delta_{0} & \text { in } \mathbf{R}^{2}, \\ u(x) \rightarrow-\infty & \text { as }|x| \rightarrow \infty,\end{cases}
$$

where the case $N=0$ is allowed. Chae-Imanuvilov [2] constructs a solution to (2.3) as a perturbation from $\log \rho_{N}$, where $\rho_{N}$ is the radially symmetric function defined by

$$
\rho_{N}(|x|)=\frac{8(N+1)^{2}|x|^{2 N}}{\left(1+|x|^{2 N+2}\right)^{2}} .
$$

Actually, it is a solution to the Liouville equation

$$
-\Delta \log \rho_{N}=\rho_{N}-4 \pi N \delta_{0} \quad \text { in } \mathbf{R}^{2} .
$$

Next, we introduce the auxiliary function $w=w_{N}(|x|) \in C^{2}\left(\mathbf{R}^{2}\right)$ by

$$
-\Delta w=\rho_{N}(x) w-\rho_{N}(x)^{2} \quad \text { in } \mathbf{R}^{2},
$$

and make a change of variables in $(2.3)$ :

$$
u(|x|)=\log \left(\varepsilon^{2} \rho_{N}(|x|)\right)+\varepsilon^{2} w_{N}(|x|)+\varepsilon^{2} v(|x|) .
$$

Then, the new unknown $v=v(|x|)$ has to satisfy

$$
-\Delta v=\frac{1}{\varepsilon^{2}} \rho_{N} e^{\varepsilon^{2}\left(v+w_{N}\right)}-\rho_{N}^{2} e^{2 \varepsilon^{2}\left(v+w_{N}\right)}-\frac{1}{\varepsilon^{2}} \rho_{N}+\Delta w_{N}
$$

in $\mathbf{R}^{2}$.

Now, we take Hilbert spaces

$$
\begin{aligned}
& X=\left\{u \in W_{l o c}^{2,2}\left(\mathbf{R}^{2}\right) \mid\|u\|_{X}^{2}=(u, u)_{X}<+\infty\right\}, \\
& Y=\left\{u \in L^{2}\left(\mathbf{R}^{2}\right) \mid\|u\|_{Y}^{2}=(u, u)_{Y}<+\infty\right\},
\end{aligned}
$$

with the inner products $(,)_{X},(,)_{Y}$ defined by

$$
\begin{aligned}
& (u, v)_{Y}=\int_{\mathbf{R}^{2}}\left(1+|x|^{2+\alpha}\right) u v d x, \\
& (u, v)_{X}=(\Delta u, \Delta v)_{Y}+\int_{\mathbf{R}^{2}} u v\left(1+|x|^{2+\alpha}\right)^{-1} d x,
\end{aligned}
$$

for $\alpha \in\left(0, \frac{1}{2}\right)$. Further, $X^{r}, Y^{r}$ denote the spaces of radially symmetric functions in $X, Y$ respectively. Then, we have the following (Lemmas 1.1 and 2.2 of [2]).

LEMMA 1. We have

$$
|v(x)| \leq\|v\|_{X}\left(\log ^{+}|x|+1\right)
$$

for $v \in X$ and $x \in \mathbf{R}^{2}$.

Lemma 2. There are $C, \tilde{C}>0$ such that

$$
\begin{aligned}
& \left|w_{N}(|x|)\right| \leq C\left(\log ^{+}|x|+1\right) \quad \text { for all } x \in \mathbf{R}^{2}, \\
& w_{N}(|x|)=-\widetilde{C} \log ^{+}|x|+o(\log |x|) \quad \text { as } \quad|x| \rightarrow \infty .
\end{aligned}
$$


In view of those lemmas, we can realize

$$
P_{N}(v, \varepsilon)=\Delta v+\frac{1}{\varepsilon^{2}} \rho_{N} e^{\varepsilon^{2}\left(v+w_{N}\right)}-\rho_{N}^{2} e^{2 \varepsilon^{2}\left(v+w_{N}\right)}-\frac{1}{\varepsilon^{2}} \rho_{N}-\rho_{N} w_{N}+\rho_{N}^{2}
$$

as a smooth mapping from a bounded neighbourhood of $(0,0)$ in $X^{r} \times \mathbf{R}$ into $Y^{r}$. Furthermore, $v \in X^{r}$ is a solution to $(2.8)$ for $\varepsilon>0$ if and only if $P_{N}(v, \varepsilon)=0$, and we have $P_{N}(0,0)=\lim _{\varepsilon \downarrow 0} P_{N}(0, \varepsilon)=0$ by the choice of $w_{N}$.

To find continuous $\varepsilon \mapsto v_{\varepsilon} \in X^{r}$ in a neighborhood of $(0,0)$ satisfying $P_{N}\left(v_{\varepsilon}, \varepsilon\right)=0$, we take the bounded linear operator

$$
L_{N}^{r}=D_{v} P_{N}(0,0)=\Delta+\rho_{N}: X^{r} \rightarrow Y^{r} .
$$

It is proven in [2] that $L_{N}^{r}$ is onto and $\operatorname{Ker}\left(L_{N}^{r}\right)$ is equal to $\operatorname{span}\left\{\phi_{N}\right\}$ for $\phi_{N}(|x|)=$ $\left(1-|x|^{2 N+2}\right) /\left(1+|x|^{2 N+2}\right)$. Therefore, taking

$$
H_{N}^{r}=\left\{u \in X^{r} \mid\left(u, \phi_{N}\right)_{X}=0\right\}=X^{r} / \operatorname{Ker} L_{N}^{r},
$$

we can apply the standard implicit function theorem and obtain $v_{\varepsilon, N}^{*} \in H_{N}^{r}$ in a neighborhood of the origin, satisfying $P_{N}\left(v_{\varepsilon, N}^{*}, \varepsilon\right)=0$ for $0<\varepsilon \ll 1$. We can check that $v_{\varepsilon, N}^{*}$ is a smooth function, and by (2.11),

$$
\left|v_{\varepsilon, N}^{*}(|x|)\right| \leq C(\varepsilon)\left(\log ^{+}|x|+1\right)
$$

with $C(\varepsilon)=\left\|v_{\varepsilon, N}^{*}\right\|_{X} \rightarrow 0$ as $\varepsilon \downarrow 0$. Thus,

$$
u_{\varepsilon, N}^{*}(|x|)=\log \left(\varepsilon^{2} \rho_{N}(|x|)\right)+\varepsilon^{2} w_{N}(|x|)+\varepsilon^{2} v_{\varepsilon, N}^{*}(|x|)
$$

is a solution to $(2.3)$ satisfying

$$
e^{u_{\varepsilon, N}^{*}}=O\left(|x|^{-2 N-4-\beta(\varepsilon)}\right)
$$

as $|x| \rightarrow \infty$ for some $\beta(\varepsilon)>0$ in $\lim _{\varepsilon \downarrow 0} \beta(\varepsilon)=0$, and hence is a "non-topological" solution.

3. Linearization. Process of glueing requires fine analysis of the linearized operator around the Chae-Imanuvilov solution, namely,

$$
A_{\varepsilon, N}=D_{v} P_{N}\left(v_{\varepsilon, N}^{*}, \varepsilon\right): X \rightarrow Y .
$$

Although the proof of this part is not described in detail in [9], we can justify the statement by using the perturbation theory for Fredholm operators. Actually, this operator is given as

$$
A_{\varepsilon, N}=\Delta+\rho_{N} e^{\varepsilon^{2}\left(w_{N}+v_{\varepsilon, N}^{*}\right)}-2 \varepsilon^{2} \rho_{N}^{2} e^{2 \varepsilon^{2}\left(w_{N}+v_{\varepsilon, N}^{*}\right)} .
$$

Because $e^{\varepsilon^{2}\left(w_{N}+v_{\varepsilon, N}^{*}\right)}=1+\varepsilon^{2} w_{N}+o\left(\varepsilon^{2}\right)$ by (2.12) and (2.14), we have

$$
A_{\varepsilon, N}=L_{N}+\varepsilon^{2} B_{N}+o\left(\varepsilon^{2}\right)
$$

in the operator norm, where

$$
L_{N}=\Delta+\rho_{N}
$$

and

$$
B_{N}=\rho_{N} w_{N}-2 \rho_{N}^{2}
$$

Now, we recall the following (Lemma 2.4, Proposition 2.2, and Lemma 2.5 of [2]). 
LEMMA 3.

$$
\operatorname{Ker} L_{N}=\operatorname{span}\left\{\phi_{N}, \phi_{N}^{+}, \phi_{N}^{-}\right\}
$$

for

$$
\begin{aligned}
\phi_{N}(x) & =\frac{1-|x|^{2 N+2}}{1+|x|^{2 N+2}} \\
\phi_{N}^{+}(x) & =\frac{|x|^{N+1} \cos (N+1) \theta}{1+|x|^{2 N+2}} \\
\phi_{N}^{-}(x) & =\frac{|x|^{N+1} \sin (N+1) \theta}{1+|x|^{2 N+2}} .
\end{aligned}
$$

LEMMA 4.

$$
\operatorname{Im} L_{N}=\left\{f \in Y \mid \int_{\mathbf{R}^{2}} f \phi_{N}^{ \pm} d x=0\right\}
$$

LEMMA 5.

$$
I_{N}^{ \pm} \equiv\left(B_{N} \phi_{N}^{ \pm}, \phi_{N}^{ \pm}\right)_{L^{2}\left(\mathbf{R}^{2}\right)} \begin{cases}<0 & (N=1,2, \cdots) \\ =0 & (N=0)\end{cases}
$$

We also make use of

$$
\left(B_{N} \phi_{N}^{+}, \phi_{N}^{-}\right)_{L^{2}\left(\mathbf{R}^{2}\right)}=\left(B_{N} \phi_{N}, \phi_{N}^{ \pm}\right)_{L^{2}\left(\mathbf{R}^{2}\right)}=0
$$

valid for $N \in \mathbf{N} \cup\{0\}$. Furthermore, we have $\phi_{N}^{ \pm} \in H_{N}$ for

$$
H_{N}=\left\{u \in X \mid\left(u, \phi_{N}\right)_{X}=0\right\},
$$

and the orthogonal decomposition $X=H_{N} \oplus H_{N}^{\perp}$ with

$$
H_{N}^{\perp}=\operatorname{span}\left\{\phi_{N}\right\} \text {. }
$$

The following lemma assures the injectivity of $\left.A_{\varepsilon, N}\right|_{H_{N}}: H_{N} \rightarrow Y$, and we provide a detailed proof for completeness. Actually, it simplifies the original one and justifies Lemma 4.2 of [9]. Let us note that (4.18) of [9] does not hold for $N=0$.

LEMmA 6. If $N \geq 1$, we have $\varepsilon_{0}>0$ and $C>0$ such that

$$
\left\|A_{\varepsilon, N} v\right\|_{Y} \geq C \varepsilon^{2}\|v\|_{X}
$$

for any $\varepsilon \in\left(0, \varepsilon_{0}\right)$ and $v \in H_{N}$.

Proof. If this is not the case, we have $\varepsilon_{n} \downarrow 0$ and $v_{n} \in H_{N}$ with $\left\|v_{n}\right\|_{X}=1$ and $\varepsilon_{n}^{-2}\left\|A_{\varepsilon, N} v_{n}\right\|_{Y} \rightarrow 0$. Then, we can extract a subsequence, denoted by the same symbol, satisfying $v_{n} \rightarrow \bar{v}$ weakly in $X$ for some $\bar{v} \in H_{N}$. This implies $A_{\varepsilon_{n}, N} v_{n} \rightarrow L_{N} \bar{v}$ weakly in $Y$, and hence $L_{N} \bar{v}=0$ follows from $\left\|A_{\varepsilon_{n}, N} v_{n}\right\|=o\left(\varepsilon_{n}^{2}\right)$. Namely, we have $\bar{v} \in \operatorname{Ker} L_{N} \cap$ $H_{N}$, and hence

$$
\bar{v}=C_{+} \phi_{N}^{+}+C_{-} \phi_{N}^{-}
$$

with some $C_{+}, C_{-} \in \mathbf{R}$ by Lemma 3 . Now, we claim that $v_{n} \rightarrow \bar{v}$ strongly in $X$, and therefore, that $\bar{v} \not \equiv 0$ or equivalently, $C_{+}^{2}+C_{-}^{2} \neq 0$. 
In fact, by (3.2) and our assumption, we have $\left\|L_{N}\left(v_{n}-\bar{v}\right)\right\|_{Y}=o(1)$, which leads to

$$
\left\|\Delta\left(v_{n}-\bar{v}\right)\right\|_{Y} \leq\left\|\rho_{N}\left(v_{n}-\bar{v}\right)\right\|_{Y}+o(1)
$$

as $n \rightarrow \infty$. On the other hand, Rellich-Kondrachov's theorem and the growth estimate (2.11) guarantee the compactness of $K_{1}=\rho_{N}: X \rightarrow Y$, and therefore, $\Delta v_{n} \rightarrow \Delta \bar{v}$ strongly in $Y$. On the other hand, $K_{2}=\left(1+|x|^{2+\alpha}\right)^{-1 / 2}: X \rightarrow L^{2}\left(\mathbf{R}^{2}\right)$ is also compact, and then we conclude that $v_{n} \rightarrow \bar{v}$ strongly in $X$ as desired.

Now, we show that this is a contradiction in the case of $N \neq 0$. In fact, for $\bar{w}=$ $\bar{v} /\left(1+|x|^{2+\alpha}\right) \in Y$ we have

$$
\frac{1}{\varepsilon_{n}^{2}}\left|\left(A_{\varepsilon_{n}, N} v_{n}, \bar{w}\right)_{Y}\right| \leq \frac{1}{\varepsilon_{n}^{2}}\left\|A_{\varepsilon_{n}, N} v_{n}\right\|_{Y}\|\bar{w}\|_{Y} \rightarrow 0
$$

while (3.2) implies

$$
\frac{1}{\varepsilon_{n}^{2}}\left(A_{\varepsilon_{n}, N} v_{n}, \bar{w}\right)_{Y}=\frac{1}{\varepsilon_{n}^{2}}\left(L_{N} v_{n}, \bar{w}\right)_{Y}+\left(B_{N} v_{n}, \bar{w}\right)_{Y}+o(1) .
$$

We also have from Lemma 4 that

$$
\frac{1}{\varepsilon_{n}^{2}}\left(L_{N} v_{n}, \bar{w}\right)_{Y}=\frac{1}{\varepsilon_{n}^{2}}\left(L_{N} v_{n}, C_{+} \phi_{N}^{+}+C_{-} \phi_{N}^{-}\right)_{L^{2}\left(\mathbf{R}^{2}\right)}=0
$$

and hence it follows that $\left(B_{N} v_{n}, \bar{w}\right)_{Y}=o(1)$. Therefore, because $v_{n} \rightarrow \bar{v}$ strongly in $X$, we have

$$
\left(B_{N} \bar{v}, \bar{w}\right)_{Y}=\left(B_{N} \bar{v}, \bar{v}\right)_{L^{2}\left(\mathbf{R}^{2}\right)}=0
$$

However,

$$
\begin{aligned}
\left(B_{N} \bar{v}, \bar{v}\right)_{L^{2}\left(\mathbf{R}^{2}\right)}= & C_{+}^{2}\left(B_{N} \phi_{N}^{+}, \phi_{N}^{+}\right)_{L^{2}\left(\mathbf{R}^{2}\right)}+C_{-}^{2}\left(B_{N} \phi_{N}^{-}, \phi_{N}^{-}\right)_{L^{2}\left(\mathbf{R}^{2}\right)} \\
& +2 C_{+} C_{-}\left(B_{N} \phi_{+, N}, \phi_{-, N}\right)_{L^{2}\left(\mathbf{R}^{2}\right)}=C_{+}^{2} I_{N}^{+}+C_{-}^{2} I_{N}^{-}<0
\end{aligned}
$$

by $N \neq 0$ and Lemma 5 , and this contradiction proves the lemma.

It is asserted in [9] that $\operatorname{Im}\left(\left.A_{\varepsilon, N}\right|_{H_{N}}\right)$ is closed in $Y$ and $\left.A_{\varepsilon, N}\right|_{H_{N}}$ is surjective if $\varepsilon>0$ sufficiently small (Lemmas 4.3 and 4.4), and therefore, $\left.A_{\varepsilon, N}\right|_{H_{N}}: H_{N} \rightarrow X$ has a bounded inverse (Lemma 4.5). We justify this by using the perturbation theory of Fredholm operators in the case of $N \geq 1$. (See [2].) Let us recall that a bounded linear operator $T: E \rightarrow F$, between Banach spaces $E, F$, is Fredholm if $\operatorname{Ker}(T)$ is of finite dimension in $E$, and $\operatorname{Im}(T)$ is closed and has a finite codimension in $F$ and that its index is defined by $\operatorname{Index}(T)=\operatorname{dim} \operatorname{Ker}(T)-\operatorname{codim} \operatorname{Im}(T)$. Then, we can make use of the following abstract theorem of Gohberg and Krein ([6]):

TheOREM 2. Let E, F be Banach spaces, and assume that the bounded linear operator $T: E \rightarrow F$ is Fredholm. Then, there is $\gamma>0$ such that if $B: E \rightarrow F$ is a bounded linear operator with $\|B\|<\gamma$, then $T+B$ is also Fredholm,

$\operatorname{dim} \operatorname{Ker}(T+B) \leq \operatorname{dim} \operatorname{Ker}(T)$,

codim $\operatorname{Im}(T+B) \leq$ codim $\operatorname{Im}(T)$,

Index $(T+B)=\operatorname{Index}(T)$. 
Actually, $B_{\varepsilon . N} \equiv A_{\varepsilon, N}-L_{N}: X \rightarrow Y$ is a bounded linear operator satisfying $\lim _{\varepsilon \downarrow 0}\left\|B_{\varepsilon, N}\right\|=0$ by (3.2). On the other hand, we have for $\left.L_{N}\right|_{H_{N}}: H_{N} \rightarrow Y$ the following.

- $\operatorname{Ker}\left(\left.L_{N}\right|_{H_{N}}\right)=\operatorname{Ker}\left(L_{N}\right) \cap H_{N}=\operatorname{span}\left\{\phi_{N}^{+}, \phi_{N}^{-}\right\}$and hence $\operatorname{dim} \operatorname{Ker}\left(\left.L_{N}\right|_{H_{N}}\right)=2$ by Lemma 3.

- We have

$$
L_{N}(X)=L_{N}\left(H_{N} \oplus H_{N}^{\perp}\right)=L_{N}\left(H_{N} \oplus \operatorname{span}\left\{\phi_{N}\right\}\right)=L_{N}\left(H_{N}\right) \oplus\{0\},
$$

and hence

$$
\operatorname{Im}\left(\left.L_{N}\right|_{H_{N}}\right)=\operatorname{Im}\left(L_{N}\right)=\left\{f \in Y \mid\left(f, \phi_{N}^{ \pm}\right)_{L^{2}\left(\mathbf{R}^{2}\right)}=0\right\}
$$

by Lemma 4 . In particular, $\operatorname{Im}\left(\left.L_{N}\right|_{H_{N}}\right)$ is closed in $Y$ and

$$
\operatorname{codim} \operatorname{Im}\left(\left.L_{N}\right|_{H_{N}}\right)=2 \text {. }
$$

Those facts guarantee that $\left.L_{N}\right|_{H_{N}}: H_{N} \rightarrow Y$ is a Fredholm operator of index 0, and we can apply Theorem 2 for $E=H_{N}, F=Y, T=\left.L_{N}\right|_{H_{N}}$, and $B=\left.B_{\varepsilon, N}\right|_{H_{N}}$. Then, we conclude that

$$
\left.A_{\varepsilon, N}\right|_{H_{N}}=\left.L_{N}\right|_{H_{N}}+\left.B_{\varepsilon, N}\right|_{H_{N}}
$$

is also a Fredholm operator of index 0 for $0<\varepsilon \ll 1$. Because Lemma 6 guarantees the injectivity of $\left.A_{\varepsilon, N}\right|_{H_{N}}: H_{N} \rightarrow Y$ for $N \geq 1$ and $0<\varepsilon \ll 1$, we get that $\left.A_{\varepsilon, N}\right|_{H_{N}}$ is also surjective then. Now, we conclude the following.

LEMmA 7. If $N \geq 1$, we have $\varepsilon_{0}>0$ such that $\left.A_{\varepsilon, N}\right|_{H_{N}}: H_{N} \rightarrow Y$ is invertible for $\varepsilon \in\left(0, \varepsilon_{0}\right)$. Furthermore, there is $C>0$ independent of $\varepsilon$ such that

$$
\left\|\left(\left.A_{\varepsilon, N}\right|_{H_{N}}\right)^{-1} u\right\|_{X} \leq \frac{C}{\varepsilon^{2}}\|u\|_{Y}
$$

for any $\varepsilon \in\left(0, \varepsilon_{0}\right)$ and $u \in Y$.

4. Glueing. Nolasco [9] constructed the approximate solution to (2.2) by "glueing" radially symmetric single vortex entire solutions to (2.5), by applying a partition of unity. More precisely, setting a false vortex point $p_{0}=0$ with multiplicity $m_{0}=0$, she studied the invertibility of the linearized operator. However, some modifications seem to be needed in this process. For example, $\left(\widehat{A}_{\varepsilon, 0}^{n}\right)^{-1}$ in $(6.34)$ of [9] does not exist.

To this end, we assume that the number of vortex points is greater than or equal to 2 , namely, $s \geq 2$. Then, we suppose that the multiplicity of $p_{1}$ is equal to

$$
m_{1}=\min _{j=1, \ldots, s}\left\{m_{j}\right\}
$$

without loss of generality, and letting $r=\frac{1}{2} \min _{i \neq j}\left\{\left|p_{i}-p_{j}\right|, \operatorname{dist}\left(p_{j}, \partial \Omega\right)\right\}$, we put

$$
\begin{aligned}
& B_{j}=\left\{x \in \Omega|| x-p_{j} \mid<r\right\}, \quad j=2, \ldots, s, \\
& B_{1}=\left\{x \in \mathbf{R}^{2} \mid \operatorname{dist}\left(x, \Omega \backslash \bigcup_{j=2, \ldots, s} B_{j}\right)<\frac{r}{2}\right\} .
\end{aligned}
$$

Let us note that $B_{i} \cap B_{j}=\emptyset$ for $i, j \in\{2, \ldots, s\}, i \neq j$, and $B_{1} \cap B_{j} \neq \emptyset$ for $j=2, \ldots, s$. By the definition, each $B_{j}$ contains exactly one vortex point $p_{j}$ with multiplicity $m_{j}$. It 
is different from [9], where $B_{0}$ contains no vortex points at all. Our situation is possible because of the assumption of $s \geq 2$.

Given $n=\left(n_{1}, n_{2}\right) \in \mathbf{Z}^{2}$ and $j=1, \ldots, s$, we set

$$
B_{j}^{n}=B_{j}+n_{1} e_{1}+n_{2} e_{2} .
$$

Then, the collection $\left\{B_{j}^{n}\right\}_{n \in \mathbf{Z}^{2}, j=1, \ldots, s}$ forms a locally finite periodic open covering of $\mathbf{R}^{2}$, and therefore, we can take an associated partition of unity

$$
\left\{\varphi_{j}^{n}\right\}_{n \in \mathbf{Z}^{2}, j=1, \ldots, s}
$$

such that $\varphi_{j}^{n} \in C_{c}^{\infty}\left(B_{j}^{n}\right), 0 \leq \varphi_{j}^{n} \leq 1$, and

$$
\sum_{n \in \mathbf{Z}^{2}} \sum_{j=1}^{s} \varphi_{j}^{n}(x)=1 \quad\left(x \in \mathbf{R}^{2}\right) .
$$

Now, we recall that the scaling parameters $0<\delta \ll \varepsilon$ are so taken as $\kappa=2 \varepsilon \delta$. Then, letting

$$
\widehat{B}_{j}^{n}=\frac{1}{\delta} B_{j}^{n}, \quad \widehat{\varphi}_{j}^{n}(x)=\varphi_{j}^{n}(\delta x)
$$

for $n \in \mathbf{Z}^{2}$ and $j=1, \ldots, s$, we have $\operatorname{supp} \widehat{\varphi}_{j}^{n} \subset \widehat{B}_{j}^{n}, 0 \leq \widehat{\varphi}_{j}^{n} \leq 1$, and

$$
\sum_{n \in \mathbf{Z}^{2}} \sum_{j=1}^{s} \widehat{\varphi}_{j}^{n}(x)=1 \quad\left(x \in \mathbf{R}^{2}\right) .
$$

That is, $\left\{\widehat{\varphi}_{j}^{n}\right\}_{n, j}$ is a partition of unity subordinate to the locally finite periodic covering $\left\{\widehat{B}_{j}^{n}\right\}_{n, j}$ of $\mathbf{R}^{2}$. We also have

$$
\sup _{x \in \hat{B}_{j}^{n}}\left|\nabla \widehat{\varphi}_{j}^{n}\right| \leq C \delta, \quad \sup _{x \in \hat{B}_{j}^{n}}\left|\Delta \widehat{\varphi}_{j}^{n}\right| \leq C \delta^{2}
$$

and

$$
\begin{array}{ll}
\widehat{B}_{i}^{n} \cap \widehat{B}_{j}^{n}=\emptyset, & i, j \in\{2, \ldots, s\}, i \neq j, \\
\widehat{B}_{1}^{n} \cap \widehat{B}_{j}^{n} \neq \emptyset, & j=2, \ldots, s, \\
\widehat{B}_{i}^{n} \cap \widehat{B}_{j}^{k}=\emptyset, & k \neq n \text { and } k \notin\langle n\rangle, i, j=1, \ldots, s, \\
\widehat{B}_{1}^{n} \cap \widehat{B}_{1}^{k} \neq \emptyset, & k \in\langle n\rangle,
\end{array}
$$

where $\langle n\rangle=\left\{k \in \mathbf{Z}^{2}|| n-k \mid=1\right\}$ denotes the nearest neighborhood of $n \in \mathbf{Z}^{2}$.

We put

$$
\widehat{\rho}_{j}^{n}(x)=\rho_{m_{j}}\left(\left|x-\widehat{p}_{j}^{n}\right|\right)=\frac{8\left(m_{j}+1\right)^{2}\left|x-\widehat{p}_{j}^{n}\right|^{2 m_{j}}}{\left(1+\left|x-\widehat{p}_{j}^{n}\right|^{2 m_{j}+2}\right)^{2}}
$$

and

$$
\widehat{w}_{j}^{n}(x)=w_{m_{j}}\left(\left|x-\widehat{p}_{j}^{n}\right|\right),
$$

where $\rho_{N}$ and $w_{N}$ stand for the functions defined by (2.4) and (2.6) for $N \in \mathbf{N} \cup\{0\}$, respectively. Further, we set

$$
\widehat{\phi_{j}^{n}}(x)=\phi_{m_{j}}\left(\left|x-\widehat{p}_{j}^{n}\right|\right)=\frac{1-\left|x-\widehat{p}_{j}^{n}\right|^{2 m_{j}+2}}{1+\left|x-\widehat{p}_{j}^{n}\right|^{2 m_{j}+2}} .
$$


We introduce Hilbert spaces

$$
\begin{aligned}
& \widehat{X}_{j}^{n}=\left\{u \in W_{l o c}^{2,2}\left(\mathbf{R}^{2}\right) \mid\|u\|_{\hat{X}_{j}^{n}}^{2}=(u, u)_{\hat{X}_{j}^{n}}<+\infty\right\}, \\
& \widehat{Y}_{j}^{n}=\left\{u \in L^{2}\left(\mathbf{R}^{2}\right) \mid\|u\|_{\hat{Y}_{j}^{n}}^{2}=(u, u)_{\hat{Y}_{j}^{n}}<+\infty\right\}, \\
& \widehat{H}_{j}^{n}=\left\{u \in \widehat{X}_{j}^{n} \mid\left(u, \widehat{\phi_{j}^{n}}\right)_{\hat{X}_{j}^{n}}=0\right\},
\end{aligned}
$$

with the inner products $(,)_{\hat{X}_{j}^{n}},(,)_{\hat{Y}_{j}^{n}}$ defined by

$$
\begin{aligned}
& (u, v)_{\hat{Y}_{j}^{n}}=\int_{\mathbf{R}^{2}}\left(1+\left(\delta\left|x-\widehat{p}_{j}^{n}\right|\right)^{2+\alpha}\right) u v \delta^{2} d x \\
& (u, v)_{\hat{X}_{j}^{n}}=(\Delta u, \Delta v)_{\hat{Y}_{j}^{n}}+\int_{\mathbf{R}^{2}} \frac{u v \delta^{2}}{1+\left(\delta\left|x-\widehat{p}_{j}^{n}\right|\right)^{2+\alpha}} d x .
\end{aligned}
$$

We also put that

$$
\begin{aligned}
& \widehat{H}_{\delta}=\left\{u \in W_{l o c}^{2,2}\left(\mathbf{R}^{2}\right) \mid \widehat{\varphi}_{j}^{n} u \in \widehat{H}_{j}^{n} \text { for any } n, j \text { and }\|u\|_{\hat{H}_{\delta}}<+\infty\right\}, \\
& \widehat{Y}_{\delta}=\left\{u \in L_{l o c}^{2}\left(\mathbf{R}^{2}\right) \mid \widehat{\varphi}_{j}^{n} u \in \widehat{Y}_{j}^{n} \text { for any } n, j \text { and }\|u\|_{\hat{Y}_{\delta}}<+\infty\right\},
\end{aligned}
$$

where

$$
\|u\|_{\hat{H}_{\delta}}=\sup _{n, j}\left\|\widehat{\varphi}_{j}^{n} u\right\|_{\hat{X}_{j}^{n}}, \quad\|u\|_{\hat{Y}_{\delta}}=\sup _{n, j}\left\|\widehat{\varphi}_{j}^{n} u\right\|_{\hat{Y}_{j}^{n}} .
$$

Finally, we take

$$
\widehat{v}_{\varepsilon, j}^{n}(x)=v_{\varepsilon, m_{j}}^{*}\left(\left|x-\widehat{p}_{j}^{n}\right|\right)
$$

for $v_{\varepsilon, N}^{*}=v_{\varepsilon, N}^{*}(|x|)$ constructed in $\S 2$, which solves $P_{N}(v, \varepsilon)=0$ for $0<\varepsilon \ll 1$. This means that $P_{j}^{n}\left(\widehat{v}_{\varepsilon, j}^{n}, \varepsilon\right)=0$ for

$$
P_{j}^{n}(v, \varepsilon)=\Delta v+\frac{1}{\varepsilon^{2}} \widehat{\rho}_{j}^{n} e^{\varepsilon^{2}\left(v+\hat{w}_{j}^{n}\right)}-\left(\widehat{\rho}_{j}^{n}\right)^{2} e^{2 \varepsilon^{2}\left(v+\hat{w}_{j}^{n}\right)}-\frac{1}{\varepsilon^{2}} \widehat{\rho}_{j}^{n}-\widehat{\rho}_{j}^{n} \widehat{w}_{j}^{n}+\left(\widehat{\rho}_{j}^{n}\right)^{2},
$$

and the linearized operator $\widehat{A}_{\varepsilon, j}^{n}=D_{v} P_{j}^{n}\left(\widehat{v}_{\varepsilon, j}^{n}, \varepsilon\right): \widehat{H}_{j}^{n} \rightarrow \widehat{Y}_{j}^{n}$, defined by

$$
\widehat{A}_{\varepsilon, j}^{n}=\Delta+\widehat{\rho}_{j}^{n} e^{\varepsilon^{2}\left(\hat{w}_{j}^{n}+\hat{v}_{\varepsilon, j}^{n}\right)}-2 \varepsilon^{2}\left(\widehat{\rho}_{j}^{n}\right)^{2} e^{2 \varepsilon^{2}\left(\hat{w}_{j}^{n}+\hat{v}_{\varepsilon, j}^{n}\right)},
$$

is subject to Lemma 7. Namely, we have the following lemma. (See Proposition 5.2 of [9].) It will become clear from this lemma that the sum of radial vortex solutions with vortex points $\widehat{p}_{j}^{n}(j=1,2, \ldots, s)$ is a good approximate solution to (2.2).

Lemma 8. There exist $\varepsilon_{0}>0$ and $C>0$ independent of $0<\delta \ll 1$ such that $\left.\widehat{A}_{\varepsilon, j}^{n}\right|_{\hat{H}_{j}^{n}}$ : $\widehat{H}_{j}^{n} \rightarrow \widehat{Y}_{j}^{n}$ is invertible and satisfies

$$
\left\|\left(\left.\widehat{A}_{\varepsilon, j}^{n}\right|_{\hat{H}_{j}^{n}}\right)^{-1} u\right\|_{\hat{X}_{j}^{n}} \leq \frac{C}{\varepsilon^{2}}\|u\|_{\hat{Y}_{j}^{n}}
$$

for any $\varepsilon \in\left(0, \varepsilon_{0}\right)$ and $u \in \widehat{Y}_{j}^{n}$.

Now, we define $z=z(x)$ by

$$
\widehat{u}(x)=\sum_{n \in \mathbf{Z}^{2}} \sum_{j=1}^{s} \widehat{\varphi}_{j}^{n}(x) \widehat{u}_{j}^{n}(x)+\varepsilon^{2} z(x)
$$

in $(2.2)$, where 


$$
\widehat{u}_{j}^{n}(x)=\log \left(\varepsilon^{2} \widehat{\rho}_{j}^{n}(x)\right)+\varepsilon^{2} \widehat{w}_{j}^{n}(x)+\varepsilon^{2} \widehat{v}_{\varepsilon, j}^{n}(x)
$$

is a solution to

$$
-\Delta \widehat{u}_{j}^{n}=\frac{1}{\varepsilon^{2}} e^{\hat{u}_{j}^{n}}\left(1-e^{\hat{u}_{j}^{n}}\right)-4 \pi m_{j} \delta_{\hat{p}_{j}^{n}}
$$

in $\mathbf{R}^{2}$. Then, $(2.2)$ is reduced to finding $z=z(x)$ satisfying

$$
\begin{aligned}
\Delta z+ & \frac{1}{\varepsilon^{2}} \sum_{n, j}\left\{\left(\Delta \widehat{\varphi}_{j}^{n}\right) \widehat{u}_{j}^{n}+2 \nabla \widehat{\varphi}_{j}^{n} \cdot \nabla \widehat{u}_{j}^{n}\right\} \\
& +\frac{1}{\varepsilon^{4}}\left(\sum_{n, j} \widehat{\varphi}_{j}^{n} e^{2 \hat{u}_{j}^{n}}-\exp \left(2 \sum_{n, j} \widehat{\varphi}_{j}^{n} \widehat{u}_{j}^{n}\right) \exp \left(2 \varepsilon^{2} z\right)\right) \\
& -\frac{1}{\varepsilon^{4}}\left(\sum_{n, j} \widehat{\varphi}_{j}^{n} e^{\hat{u}_{j}^{n}}-\exp \left(\sum_{n, j} \widehat{\varphi}_{j}^{n} \widehat{u}_{j}^{n}\right) \exp \left(\varepsilon^{2} z\right)\right)=0
\end{aligned}
$$

in $\mathbf{R}^{2}$, because $\sum_{n, j} \widehat{\varphi}_{j}^{n} m_{j} \delta_{\hat{p}_{j}^{n}}-\sum_{n, j} m_{j} \delta_{\hat{p}_{j}^{n}} \equiv 0$. In the rest of this section, we shall represent (4.15) in a simple form.

First, we set

$$
C_{j}^{n}(x)=\left(\Delta \widehat{\varphi}_{j}^{n}\right) \widehat{u}_{j}^{n}+2 \nabla \widehat{\varphi}_{j}^{n} \cdot \nabla \widehat{u}_{j}^{n}
$$

for $n \in \mathbf{Z}^{2}, j=1, \ldots, s$. Next, we put

$$
\begin{aligned}
& R_{1}^{n}(x) \equiv \exp \left\{\sum_{l=2}^{s} \widehat{\varphi}_{l}^{n}\left(\widehat{u}_{l}^{n}-\widehat{u}_{1}^{n}\right)\right\} \times \exp \left\{\sum_{k \in\langle n\rangle} \widehat{\varphi}_{1}^{k}\left(\widehat{u}_{1}^{k}-\widehat{u}_{1}^{n}\right)\right\} \\
& =\exp \left\{\sum_{l=2}^{s} \widehat{\varphi}_{l}^{n}\left(\log \frac{\widehat{\rho}_{l}^{n}}{\widehat{\rho}_{1}^{n}}+\varepsilon^{2}\left(\widehat{w}_{l}^{n}-\widehat{w}_{1}^{n}\right)+\varepsilon^{2}\left(\widehat{v}_{\varepsilon, l}^{n}-\widehat{v}_{\varepsilon, 1}^{n}\right)\right)\right\} \\
& \quad \times \exp \left\{\sum_{k \in\langle n\rangle} \widehat{\varphi}_{1}^{k}\left(\log \frac{\widehat{\rho}_{1}^{k}}{\widehat{\rho}_{1}^{n}}+\varepsilon^{2}\left(\widehat{w}_{1}^{k}-\widehat{w}_{1}^{n}\right)+\varepsilon^{2}\left(\widehat{v}_{\varepsilon, 1}^{k}-\widehat{v}_{\varepsilon, 1}^{n}\right)\right)\right\}, \\
& R_{j}^{n}(x) \equiv \exp \left\{\widehat{\varphi}_{1}^{n}\left(\widehat{u}_{1}^{n}-\widehat{u}_{j}^{n}\right)\right\} \\
& =\exp \left\{\widehat{\varphi}_{1}^{n}\left(\log \frac{\widehat{\rho}_{1}^{n}}{\widehat{\rho}_{j}^{n}}+\varepsilon^{2}\left(\widehat{w}_{1}^{n}-\widehat{w}_{j}^{n}\right)+\varepsilon^{2}\left(\widehat{v}_{\varepsilon, 1}^{n}-\widehat{v}_{\varepsilon, j}^{n}\right)\right)\right\},
\end{aligned}
$$

for $j=2, \ldots, s$. Then, we obtain

$$
\begin{array}{ll}
R_{1}^{n}(x)=1 \quad \text { on } \quad\left(\bigcup_{l=2}^{s} \widehat{B}_{l}^{n} \cup \bigcup_{k \in\langle n\rangle} \widehat{B}_{1}^{k}\right)^{c}, \\
R_{j}^{n}(x)=1 \quad \text { on } \quad\left(\widehat{B}_{1}^{n}\right)^{c}, \quad j=2, \ldots, s .
\end{array}
$$

On the other hand, we have from (4.3) that

$$
\sum_{n, j} \widehat{\varphi}_{j}^{n} \widehat{u}_{j}^{n}=\left\{\begin{array}{l}
\widehat{\varphi}_{1}^{n} \widehat{u}_{1}^{n}+\widehat{\varphi}_{j}^{n} \widehat{u}_{j}^{n} \quad\left(x \in \widehat{B}_{j}^{n}, j=2, \ldots, s\right) \\
\sum_{l=1}^{s} \widehat{\varphi}_{l}^{n} \widehat{u}_{l}^{n}+\sum_{k \in\langle n\rangle} \widehat{\varphi}_{1}^{k} \widehat{u}_{1}^{k} \quad\left(x \in \widehat{B}_{1}^{n}\right)
\end{array}\right.
$$

Now

$$
\exp \left(\sum_{n, j} \widehat{\varphi}_{j}^{n} \widehat{u}_{j}^{n}\right)=R_{j}^{n}(x) e^{\hat{u}_{j}^{n}(x)}=\varepsilon^{2} \widehat{\rho}_{j}^{n} e^{\varepsilon^{2}\left(\hat{w}_{j}^{n}+\hat{v}_{\varepsilon, j}^{n}\right)} R_{j}^{n} \quad \text { on } \quad \widehat{B}_{j}^{n}
$$


by (4.17) and (4.18), where $n \in \mathbf{Z}^{2}, j=1, \ldots, s$. In fact, if $x \in \widehat{B}_{j}^{n}$ for $j \neq 1$, we have $\widehat{\varphi}_{1}^{n}(x)+\widehat{\varphi}_{j}^{n}(x)=1$, and hence

$$
\exp \left(\sum_{n, j} \widehat{\varphi}_{j}^{n} \widehat{u}_{j}^{n}\right)=e^{\hat{\varphi}_{1}^{n} \hat{u}_{1}^{n}+\hat{\varphi}_{j}^{n} \hat{u}_{j}^{n}}=e^{\hat{\varphi}_{1}^{n} \hat{u}_{1}^{n}-\hat{\varphi}_{1}^{n} \hat{u}_{j}^{n}} \cdot e^{\left(\hat{\varphi}_{1}^{n}+\hat{\varphi}_{j}^{n}\right) \hat{u}_{j}^{n}}=R_{j}^{n}(x) e^{\hat{u}_{j}^{n}} .
$$

For $x \in \widehat{B}_{1}^{n}$, we have $\sum_{l=1}^{s} \widehat{\varphi}_{l}^{n}(x)+\sum_{k \in\langle n\rangle} \widehat{\varphi}_{1}^{k}(x)=1$, and therefore,

$$
\begin{aligned}
\exp \left(\sum_{n, j} \widehat{\varphi}_{j}^{n} \widehat{u}_{j}^{n}\right)= & \exp \left(\sum_{l=1}^{s} \widehat{\varphi}_{l}^{n} \widehat{u}_{l}^{n}+\sum_{k \in<n>} \widehat{\varphi}_{1}^{k} \widehat{u}_{1}^{k}\right) \\
= & \exp \left(\sum_{l=1}^{s} \widehat{\varphi}_{l}^{n}\left(\widehat{u}_{l}^{n}-\widehat{u}_{1}^{n}\right)+\sum_{k \in<n>} \widehat{\varphi}_{1}^{k}\left(\widehat{u}_{1}^{k}-\widehat{u}_{1}^{n}\right)\right) \\
& \times \exp \left(\left(\sum_{l=1}^{s} \widehat{\varphi}_{l}^{n}+\sum_{k \in<n>} \widehat{\varphi}_{1}^{k}\right) \widehat{u}_{1}^{n}\right)=R_{1}^{n}(x) e^{\hat{u}_{1}^{n}} .
\end{aligned}
$$

This proves (4.19).

By (4.19), we have

$$
\exp \left(\sum_{n, j} \widehat{\varphi}_{j}^{n} \widehat{u}_{j}^{n}\right)=\sum_{n, j} \widehat{\varphi}_{j}^{n} R_{j}^{n} e^{\hat{u}_{j}^{n}}, \quad \exp \left(2 \sum_{n, j} \widehat{\varphi}_{j}^{n} \widehat{u}_{j}^{n}\right)=\sum_{n, j} \widehat{\varphi}_{j}^{n}\left(R_{j}^{n}\right)^{2} e^{\hat{u}_{j}^{n}}
$$

on $\mathbf{R}^{2}$. Therefore, (4.15) is equivalent to

$$
F_{\varepsilon, \delta}(z)=0
$$

where

$$
\begin{aligned}
& F_{\varepsilon, \delta}(z)=\Delta z+\frac{1}{\varepsilon^{2}} \sum_{n, j} C_{j}^{n}(x) \\
& +\frac{1}{\varepsilon^{2}} \sum_{n, j} \widehat{\varphi}_{j}^{n} \widehat{\rho}_{j}^{n} e^{\varepsilon^{2}\left(\hat{w}_{j}^{n}+\hat{v}_{\varepsilon, j}^{n}\right)}\left(e^{\varepsilon^{2} z} R_{j}^{n}(x)-1\right) \\
& -\frac{1}{\varepsilon^{2}} \sum_{n, j} \widehat{\varphi}_{j}^{n}\left(\widehat{\rho}_{j}^{n}\right)^{2} e^{2 \varepsilon^{2}\left(\hat{w}_{j}^{n}+\hat{v}_{\varepsilon, j}^{n}\right)}\left(e^{2 \varepsilon^{2} z} R_{j}^{n}(x)^{2}-1\right) .
\end{aligned}
$$

This equation is solved by Banach's fixed point theorem, and in the next section we study the linearized operator.

5. Second linearization. We show the solvability of $F_{\varepsilon, \delta}(z)=0$ by examining the method of [9] in detail. First, $F_{\varepsilon, \delta}: \widehat{H}_{\delta} \rightarrow \widehat{Y}_{\delta}$ is well defined by (2.12) and (2.14). More precisely, we have the following estimates (Lemmas 6.2, 6.3, 6.4 of [9]).

LEMMA 9. We have

$$
\sup _{x \in \mathbf{R}^{2}} \widehat{\varphi}_{j}^{n}(x) \widehat{\rho}_{j}^{n}(x)\left|R_{j}^{n}(x)-1\right|=O\left(\delta^{4-\beta(\varepsilon)}\right)
$$

and

$$
\left\|\sum_{n, j} C_{j}^{n}(x)\right\|_{\hat{Y}_{\delta}}=O\left(\delta^{2}|\log \delta|\right)
$$

as $\delta \downarrow 0$ with $\beta(\varepsilon) \rightarrow 0$, where $n \in \mathbf{Z}^{2}$ and $j=1, \ldots, s$. 
Proof. By (4.5) and

$$
\operatorname{diam}\left(\widehat{B}_{j}^{n}\right)=O\left(\delta^{-1}\right)
$$

there are $C_{1}, C_{2}>0$ such that

$$
C_{1} \delta^{2 m_{j}+4} \leq \widehat{\rho}_{j}^{n}(x) \leq C_{2} \delta^{2 m_{j}+4},
$$

where $n \in \mathbf{Z}^{2}, j=1, \ldots, s$ and $x \in \widehat{B}_{j}^{n}$. Hence

$$
\begin{aligned}
& \sup _{x \in \hat{B}_{j}^{n} \cap \hat{B}_{1}^{n}} \frac{\widehat{\rho}_{j}^{n}(x)}{\widehat{\rho}_{1}^{n}(x)} \leq C \delta^{2 m_{j}-2 m_{1}} \leq C \quad(j=2, \ldots, s), \\
& \sup _{x \in \hat{B}_{1}^{k} \cap \hat{B}_{1}^{n}} \frac{\widehat{\rho}_{1}^{k}(x)}{\widehat{\rho}_{1}^{n}(x)} \leq C \quad(k \in\langle n\rangle) .
\end{aligned}
$$

In fact, the exponent of $\delta$ in (5.4) is nonnegative by our choice of $m_{1}$. Moreover by (2.12) and (2.14), we have

$$
\sup _{x \in \hat{B}_{j}^{n} \cap \hat{B}_{1}^{n}}\left(\left|\widehat{w}_{1}^{n}(x)-\widehat{w}_{j}^{n}(x)\right|+\left|\widehat{v}_{\varepsilon, 1}^{n}(x)-\widehat{v}_{\varepsilon, j}^{n}(x)\right|\right) \leq C|\log \delta|
$$

for $j=2, \ldots, s$ and

$$
\sup _{x \in \hat{B}_{1}^{k} \cap \hat{B}_{1}^{n}}\left(\left|\widehat{w}_{1}^{n}(x)-\widehat{w}_{1}^{k}(x)\right|+\left|\widehat{v}_{\varepsilon, 1}^{n}(x)-\widehat{v}_{\varepsilon, 1}^{k}(x)\right|\right) \leq C|\log \delta|
$$

for $k \in\langle n\rangle$. Therefore by (5.4) and (4.17), we have

$$
\left|R_{j}^{n}(x)\right| \leq \exp \left(C+C \varepsilon^{2}|\log \delta|\right)=C \delta^{-C \varepsilon^{2}}
$$

for all $x \in \mathbf{R}^{2}$. This implies that

$$
\sup _{x \in \mathbf{R}^{2}} \widehat{\varphi}_{j}^{n}(x) \widehat{\rho}_{j}^{n}(x)\left|R_{j}^{n}(x)-1\right| \leq \sup _{x \in \hat{B}_{j}^{n}} \widehat{\rho}_{j}^{n}(x)\left(R_{j}^{n}(x)+1\right) \leq C \delta^{2 m_{j}+4-\beta(\varepsilon)}
$$

for $0<\delta \ll 1$, where $\beta(\varepsilon)=C \varepsilon^{2}$. Thus, we obtain (5.1).

Now, we note the following: If $f_{\ell}^{k}\left(k \in \mathbf{Z}^{2}, \ell \in\{1, \ldots, s\}\right)$ are functions satisfying $\operatorname{supp}\left(f_{\ell}^{k}\right) \subset \widehat{B}_{l}^{k}$, then

$$
\left\|\sum_{k, l} f_{\ell}^{k}\right\|_{\hat{Y}_{\delta}} \leq C \sup _{k, \ell}\left\|f_{\ell}^{k}\right\|_{\hat{Y}_{l}^{k}}
$$

where $C$ is a constant independent of $k, \ell$. In fact,

$$
\left\|\sum_{k, l} f_{\ell}^{k}\right\|_{\hat{Y}_{\delta}}=\sup _{n, j}\left\|\widehat{\varphi}_{j}^{n} \sum_{k, l} f_{\ell}^{k}\right\|_{\hat{Y}_{j}^{n}} \leq C \sup _{n, j} \sup _{\substack{k, \ell \\ \hat{B}_{j}^{n} \cap \hat{B}_{l}^{k} \neq \emptyset}}\left\|\widehat{\varphi}_{j}^{n} f_{\ell}^{k}\right\|_{\hat{Y}_{j}^{n}}
$$

and

$$
\begin{aligned}
\left\|\widehat{\varphi}_{j}^{n} f_{\ell}^{k}\right\|_{\hat{Y}_{j}^{n}} & =\int_{\mathbf{R}^{2}}\left(1+\left(\delta\left|x-\widehat{p}_{j}^{n}\right|\right)^{2+\alpha}\right)\left|\widehat{\varphi}_{j}^{n}(x) f_{\ell}^{k}(x)\right|^{2} \delta^{2} d x \\
& =\int_{\hat{B}_{j}^{n} \cap \hat{B}_{l}^{k}} \frac{1+\left(\delta\left|x-\widehat{p}_{j}^{n}\right|\right)^{2+\alpha}}{1+\left(\delta\left|x-\widehat{p}_{l}^{k}\right|\right)^{2+\alpha}}\left(1+\left(\delta\left|x-\widehat{p}_{l}^{k}\right|\right)^{2+\alpha}\right)\left|\widehat{\varphi}_{j}^{n} f_{l}^{k}\right|^{2} \delta^{2} d x \\
& \leq C \int_{\hat{B}_{l}^{k}}\left(1+\left(\delta\left|x-\widehat{p}_{l}^{k}\right|\right)^{2+\alpha}\right)\left|f_{l}^{k}(x)\right|^{2} \delta^{2} d x
\end{aligned}
$$


because

$$
\frac{1+\left(\delta\left|x-\widehat{p}_{j}^{n}\right|\right)^{2+\alpha}}{1+\left(\delta\left|x-\widehat{p}_{l}^{k}\right|\right)^{2+\alpha}}=O(1) \quad\left(x \in \widehat{B}_{j}^{n} \cap \widehat{B}_{l}^{k}\right)
$$

and $\widehat{\varphi}_{j}^{n}(x) \leq 1$. This shows $(5.6)$.

From this, we obtain that

$$
\begin{aligned}
\left\|\sum_{n, j} C_{j}^{n}\right\|_{\hat{Y}_{\delta}} & \leq C \sup _{n, j}\left\|C_{j}^{n}\right\|_{\hat{Y}_{j}^{n}} \\
& \leq C \sup _{x \in \hat{B}_{j}^{n}}\left(\left|\Delta \widehat{\varphi}_{j}^{n}(x)\left\|\widehat{u}_{j}^{n}(x)|+2| \nabla \widehat{\varphi}_{j}^{n}(x)\right\| \nabla \widehat{u}_{j}^{n}(x)\right|\right) .
\end{aligned}
$$

Similarly, we have from $(2.11),(2.12),(2.14)$ that

$$
\left|\widehat{u}_{j}^{n}(x)\right| \leq C|\log | x-\widehat{p}_{j}^{n}||, \quad\left|\nabla \widehat{u}_{j}^{n}(x)\right| \leq \frac{C}{\left|x-\widehat{p}_{j}^{n}\right|}
$$

as $|x| \rightarrow \infty$, where $C>0$ is a constant independent of $n, j$. We also have (4.2), and hence (5.2) follows as

$$
\left\|\sum_{n, j} C_{j}^{n}(x)\right\|_{\hat{Y}_{\delta}} \leq C\left(\delta^{2} \log \frac{1}{\delta}+\delta^{2}\right)
$$

The proof is complete.

Now, we see that $F_{\varepsilon, \delta}$ is a smooth map from the unit ball of $\widehat{H}_{\delta}$ to $\widehat{Y}_{\delta}$ if $0<\varepsilon \ll 1$. Furthermore, by (2.12), (2.14) and (5.3), we have

$$
\sup _{x \in \hat{B}_{j}^{n}} e^{\varepsilon^{2}\left(\hat{w}_{j}^{n}+\hat{v}_{\varepsilon, j}^{n}\right)} \leq C \delta^{-C \varepsilon^{2}},
$$

and hence

$$
\begin{aligned}
\| \widehat{\varphi}_{j}^{n} \widehat{\rho}_{j}^{n} e^{\varepsilon^{2}\left(\hat{w}_{j}^{n}+\hat{v}_{\varepsilon, j}^{n}\right)\left[R_{j}^{n}-1\right] \|_{\hat{Y}_{j}^{n}}} \leq \sup _{x \in \hat{B}_{j}^{n}} \widehat{\varphi}_{j}^{n}(x) \widehat{\rho}_{j}^{n}(x) e^{\varepsilon^{2}\left(\hat{w}_{j}^{n}+\hat{v}_{\varepsilon, j}^{n}\right)}\left|R_{j}^{n}(x)-1\right| \\
\leq C \delta^{2 m_{j}+4-C \varepsilon^{2}}=o\left(\delta^{2}\right)
\end{aligned}
$$

as $\delta \downarrow 0$. Similarly, we have

$$
\left\|\widehat{\varphi}_{j}^{n}\left(\widehat{\rho}_{j}^{n}\right)^{2} e^{2 \varepsilon^{2}\left(\hat{w}_{j}^{n}+\hat{v}_{\varepsilon, j}^{n}\right)}\left[\left(R_{j}^{n}\right)^{2}-1\right]\right\|_{\hat{Y}_{j}^{n}} \leq o\left(\delta^{2}\right) .
$$

Therefore, Lemma 9 guarantees the following.

LEMMA 10. For $0<\delta \ll \varepsilon \ll 1$,

$$
\left\|F_{\varepsilon, \delta}(0)\right\|_{\hat{Y}_{\delta}} \leq \frac{C}{\varepsilon^{2}} \delta^{2}|\log \delta|
$$

as $\delta \downarrow 0$.

Now, we turn to $A_{\varepsilon, \delta} \equiv D F_{\varepsilon, \delta}(0): \widehat{H}_{\delta} \rightarrow \widehat{Y}_{\delta}$. It is realized as

$$
A_{\varepsilon, \delta}=\Delta+\sum_{n, j} \widehat{\varphi}_{j}^{n} \widehat{\rho}_{j}^{n} e^{\varepsilon^{2}\left(\hat{w}_{j}^{n}+\hat{v}_{\varepsilon, j}^{n}\right)} R_{j}^{n}(x)-2 \varepsilon^{2} \sum_{n, j} \widehat{\varphi}_{j}^{n}\left(\widehat{\rho}_{j}^{n}\right)^{2} e^{2 \varepsilon^{2}\left(\hat{w}_{j}^{n}+\hat{v}_{\varepsilon, j}^{n}\right)} R_{j}^{n}(x)^{2} .
$$

The next lemma is Lemma 6.4 of [9]. There, it is shown that this operator is approximated locally by $\widehat{A}_{\varepsilon, j}^{n}$ of $(4.11)$ :

$$
\widehat{A}_{\varepsilon, j}^{n}=\Delta+\widehat{\rho}_{j}^{n} e^{\varepsilon^{2}\left(\hat{w}_{j}^{n}+\hat{v}_{\varepsilon, j}^{n}\right)}-2 \varepsilon^{2}\left(\widehat{\rho}_{j}^{n}\right)^{2} e^{2 \varepsilon^{2}\left(\hat{w}_{j}^{n}+\hat{v}_{\varepsilon, j}^{n}\right)} .
$$


LEMmA 11. There are $C>0$ and $\beta(\varepsilon) \rightarrow 0$ as $\varepsilon \downarrow 0$ such that

$$
\left\|\left(A_{\varepsilon, \delta}-\widehat{A}_{\varepsilon, j}^{n}\right)\left(\widehat{\varphi}_{j}^{n} h\right)\right\|_{\hat{Y}_{j}^{n}} \leq C \delta^{4-\beta(\varepsilon)}\left\|\widehat{\varphi}_{j}^{n} h\right\|_{\hat{X}_{j}^{n}}
$$

for any $n, j$ and $h \in \widehat{H}_{\delta}$ in the case of $0<\delta \ll \varepsilon \ll 1$.

Proof. In fact, we have

$$
\begin{aligned}
& \left\|\left(A_{\varepsilon, \delta}-\widehat{A}_{\varepsilon, j}^{n}\right)\left(\widehat{\varphi}_{j}^{n} h\right)\right\|_{\hat{Y}_{j}^{n}} \leq\left\|\hat{\rho}_{j}^{n} e^{\varepsilon^{2}\left(\hat{w}_{j}^{n}+\hat{v}_{\varepsilon, j}^{n}\right)}\left(\widehat{\varphi}_{j}^{n} R_{j}^{n}(x)-1\right)\left(\widehat{\varphi}_{j}^{n} h\right)\right\|_{\hat{Y}_{j}^{n}} \\
& +2 \varepsilon^{2}\left\|\left(\widehat{\rho}_{j}^{n}\right)^{2} e^{2 \varepsilon^{2}\left(\hat{w}_{j}^{n}+\hat{v}_{\varepsilon, j}^{n}\right)}\left(\widehat{\varphi}_{j}^{n} R_{j}^{n}(x)^{2}-1\right)\left(\widehat{\varphi}_{j}^{n} h\right)\right\|_{\hat{Y}_{j}^{n}} \\
& +\left\|\sum_{(k, l) \neq(n, j)} \widehat{\varphi}_{l}^{k} \widehat{\rho}_{l}^{k} e^{\varepsilon^{2}\left(\hat{w}_{l}^{k}+\hat{v}_{\varepsilon, l}^{k}\right)} R_{l}^{k}(x)\left(\widehat{\varphi}_{j}^{n} h\right)\right\|_{\hat{Y}_{j}^{n}} \\
& +2 \varepsilon^{2}\left\|\sum_{(k, l) \neq(n, j)} \widehat{\varphi}_{l}^{k}\left(\widehat{\rho}_{l}^{k}\right)^{2} e^{2 \varepsilon^{2}\left(\hat{w}_{l}^{k}+\hat{v}_{\varepsilon, l}^{k}\right)} R_{l}^{k}(x)^{2}\left(\widehat{\varphi}_{j}^{n} h\right)\right\|_{\hat{Y}_{j}^{n}} .
\end{aligned}
$$

We apply (5.9) and (5.1) for the first term of the right-hand side, and get that

$$
\begin{aligned}
\| \hat{\rho}_{j}^{n} e^{\varepsilon^{2}\left(\hat{w}_{j}^{n}+\hat{v}_{\varepsilon, j}^{n}\right)} & \left(\widehat{\varphi}_{j}^{n} R_{j}^{n}(x)-1\right)\left(\widehat{\varphi}_{j}^{n} h\right) \|_{\hat{Y}_{j}^{n}} \\
\leq & \sup _{x \in \hat{B}_{j}^{n}}\left(\widehat{\rho}_{j}^{n}(x) e^{\varepsilon^{2}\left(\hat{w}_{j}^{n}+\hat{v}_{\varepsilon, j}^{n}\right)}\left|R_{j}^{n}(x)-1\right|\right)\left\|\widehat{\varphi}_{j}^{n} h\right\|_{\hat{Y}_{j}^{n}} \leq C \delta^{4-\beta(\varepsilon)}\left\|\widehat{\varphi}_{j}^{n} h\right\|_{\hat{X}_{j}^{n}}
\end{aligned}
$$

Similarly, we have

$$
\left\|\left(\widehat{\rho}_{j}^{n}\right)^{2} e^{2 \varepsilon^{2}\left(\hat{w}_{j}^{n}+\hat{v}_{\varepsilon, j}^{n}\right)}\left(\widehat{\varphi}_{j}^{n} R_{j}^{n}(x)^{2}-1\right)\left(\widehat{\varphi}_{j}^{n} h\right)\right\|_{\hat{Y}_{j}^{n}} \leq o\left(\delta^{4-\beta(\varepsilon)}\right)\left\|\widehat{\varphi}_{j}^{n} h\right\|_{\hat{X}_{j}^{n}} .
$$

For the third and the fourth terms, we apply (5.6) and (5.9). Then, we get that

$$
\begin{aligned}
& \left\|\sum_{(k, l) \neq(n, j)} \widehat{\varphi}_{l}^{k} \widehat{\rho}_{l}^{k} e^{\varepsilon^{2}\left(\hat{w}_{l}^{k}+\hat{v}_{\varepsilon, l}^{k}\right)} R_{l}^{k}(x)\left(\widehat{\varphi}_{j}^{n} h\right)\right\|_{\hat{Y}_{j}^{n}} \\
& \quad \leq \sup _{(k, l) \neq(n, j)} \sup _{x \in \hat{B}_{j}^{n} \cap \hat{B}_{l}^{k}} \widehat{\rho}_{l}^{k}(x) e^{\varepsilon^{2}\left(\hat{w}_{l}^{k}+\hat{v}_{\varepsilon, l}^{k}\right)} R_{l}^{k}(x)\left\|\widehat{\varphi}_{j}^{n} h\right\|_{\hat{Y}_{j}^{n}} \leq C \delta^{4-\beta(\varepsilon)}\left\|\widehat{\varphi}_{j}^{n} h\right\|_{\hat{X}_{j}^{n}},
\end{aligned}
$$

and

$$
\left\|\sum_{(k, l) \neq(n, j)} \widehat{\varphi}_{l}^{k}\left(\widehat{\rho}_{l}^{k}\right)^{2} e^{2 \varepsilon^{2}\left(\hat{w}_{l}^{k}+\hat{v}_{\varepsilon, l}^{k}\right)} R_{l}^{k}(x)^{2}\left(\widehat{\varphi}_{j}^{n} h\right)\right\|_{\hat{Y}_{j}^{n}} \leq o\left(\delta^{4-\beta(\varepsilon)}\right)\left\|\widehat{\varphi}_{j}^{n} h\right\|_{\hat{X}_{j}^{n}} .
$$

From these estimates, we obtain the lemma.

Now, we show the main result of section. (See Proposition 6.5 of [9].)

TheOREm 3. Given $0<\varepsilon \ll 1$, there exists $\delta=\delta_{\varepsilon}>0$ such that $A_{\varepsilon, \delta}=D F_{\varepsilon, \delta}(0)$ : $\widehat{H}_{\delta} \rightarrow \widehat{Y}_{\delta}$ is invertible for any $\delta \in\left(0, \delta_{\varepsilon}\right)$. Moreover, $\left\|A_{\varepsilon, \delta}^{-1}\right\| \leq C / \varepsilon^{2}$ with a constant $C>0$ independent of $\delta \in\left(0, \delta_{\varepsilon}\right)$ and $0<\varepsilon \ll 1$.

Proof. We follow the key idea of the original proof [9], and take the smooth function

$$
\widehat{g}_{j}^{n}(x)=\frac{\widehat{\varphi}_{j}^{n}(x)}{\left\{\sum_{k, l} \widehat{\varphi}_{l}^{k}(x)^{2}\right\}^{1 / 2}},
$$


where $n \in \mathbf{Z}^{2}$ and $j=1, \ldots, s$. Now, we introduce the auxiliary linear operator $S_{\varepsilon, \delta}$ : $\widehat{H}_{\delta} \rightarrow \widehat{Y}_{\delta}$ by

$$
S_{\varepsilon, \delta} h=\sum_{n \in \mathbf{Z}^{2}} \sum_{j=1, \ldots, s} \widehat{g}_{j}^{n}\left(\widehat{A}_{\varepsilon, j}^{n}\right)^{-1}\left(\widehat{g}_{j}^{n} h\right)
$$

where $h \in \widehat{Y}_{\delta}$. In fact, we have from (5.6) and Lemma 8 that

$$
\left\|S_{\varepsilon, \delta} h\right\|_{\hat{H}_{\delta}} \leq C \sup _{n, j}\left\|\widehat{g}_{j}^{n}\left(\widehat{A}_{\varepsilon, j}^{n}\right)^{-1} \widehat{g}_{j}^{n}\right\| \leq \frac{C}{\varepsilon^{2}}\|h\|_{\hat{Y}_{\delta}},
$$

with a constant $C>0$ independent of $\delta$ in $0<\delta \ll \varepsilon$.

We shall show that both $A_{\varepsilon, \delta} S_{\varepsilon, \delta}: \widehat{Y}_{\delta} \rightarrow \widehat{Y}_{\delta}$ and $S_{\varepsilon, \delta} A_{\varepsilon, \delta}: \widehat{H}_{\delta} \rightarrow \widehat{H}_{\delta}$ are invertible. In fact, we have

$$
S_{\varepsilon, \delta} A_{\varepsilon, \delta}=I_{\hat{H}_{\delta}}+\sum_{n, j} \widehat{g}_{j}^{n}\left(\widehat{A}_{\varepsilon, j}^{n}\right)^{-1}\left(A_{\varepsilon, \delta}-\widehat{A}_{\varepsilon, j}^{n}\right) \widehat{g}_{j}^{n}-\sum_{n, j} \widehat{g}_{j}^{n}\left(\widehat{A}_{\varepsilon, j}^{n}\right)^{-1}\left[A_{\varepsilon, \delta}, \widehat{g}_{j}^{n}\right],
$$

with the commutator $\left[A_{\varepsilon, \delta}, \widehat{g}_{j}^{n}\right]$ given by

$$
\left[A_{\varepsilon, \delta}, \widehat{g}_{j}^{n}\right] h=A_{\varepsilon, \delta}\left(\widehat{g}_{j}^{n} h\right)-\widehat{g}_{j}^{n} A_{\varepsilon, \delta} h=\Delta\left(\widehat{g}_{j}^{n} h\right)-\widehat{g}_{j}^{n} \Delta h=\left[\Delta, \widehat{g}_{j}^{n}\right] h
$$

for $h \in \widehat{H}_{\delta}$. Then, we can prove that the error term $S_{\varepsilon, \delta} A_{\varepsilon, \delta}-I_{\hat{H}_{\delta}}$ in (5.16) is small in the operator norm for $0<\delta \ll \varepsilon$. Because the original proof of [9] is not described in detail at this stage, here we examine it in full length.

First, by (5.6) and Lemmas 8 and 11, we have

$$
\begin{aligned}
\| \sum_{n, j} \widehat{g}_{j}^{n}\left(\widehat{A}_{\varepsilon, j}^{n}\right)^{-1} & \left(A_{\varepsilon, \delta}-\widehat{A}_{\varepsilon, j}^{n}\right)\left(\widehat{g}_{j}^{n} h\right) \|_{\hat{H}_{\delta}} \\
& \leq C \sup _{n, j}\left\|\widehat{g}_{j}^{n}\left(\widehat{A}_{\varepsilon, j}^{n}\right)^{-1}\left(A_{\varepsilon, \delta}-\widehat{A}_{\varepsilon, j}^{n}\right)\left(\widehat{g}_{j}^{n} h\right)\right\|_{\hat{X}_{j}^{n}} \\
& \leq \frac{C}{\varepsilon^{2}} \sup _{n, j}\left\|\left(A_{\varepsilon, \delta}-\widehat{A}_{\varepsilon, j}^{n}\right)\left(\widehat{\varphi}_{j}^{n} h\right)\right\|_{\hat{Y}_{j}^{n}} \leq \frac{C}{\varepsilon^{2}} \delta^{4-\beta(\varepsilon)} \sup _{n, j}\left\|\widehat{\varphi}_{j}^{n} h\right\|_{\hat{X}_{j}^{n}}
\end{aligned}
$$

for any $h \in \widehat{H}_{\delta}$. Similarly, we have

$$
\begin{aligned}
\left\|\sum_{n, j} \widehat{g}_{j}^{n}\left(\widehat{A}_{\varepsilon, j}^{n}\right)^{-1}\left[A_{\varepsilon, \delta}, \widehat{g}_{j}^{n}\right] h\right\|_{\hat{H}_{\delta}} & \leq C \sup _{n, j}\left\|\widehat{g}_{j}^{n}\left(\widehat{A}_{\varepsilon, j}^{n}\right)^{-1}\left[A_{\varepsilon, \delta}, \widehat{g}_{j}^{n}\right] h\right\|_{\hat{X}_{j}^{n}} \\
& \leq \frac{C}{\varepsilon^{2}} \sup _{n, j}\left\|\left[\Delta, \widehat{g}_{j}^{n}\right] h\right\|_{\hat{Y}_{j}^{n}} .
\end{aligned}
$$

Now, we shall show that

$$
\left\|\left[\Delta, \widehat{g}_{j}^{n}\right] h\right\|_{\hat{Y}_{j}^{n}}=O(\delta) \sup _{n, j}\left\|\widehat{\varphi}_{j}^{n} h\right\|_{\hat{X}_{j}^{n}}
$$

as $\delta \downarrow 0$. Actually, this part requires several (rather delicate) modifications of the original paper. First, we note

$$
\sup _{x \in \hat{B}_{j}^{n}}\left|\Delta \widehat{g}_{j}^{n}(x)\right| \leq C \delta^{2}, \quad \sup _{x \in \hat{B}_{j}^{n}}\left|\nabla \widehat{g}_{j}^{n}(x)\right| \leq C \delta,
$$

and $1+\left(\delta\left|x-\widehat{p}_{j}^{n}\right|\right)^{2+\alpha}=O(1)$ for $x \in \widehat{B}_{j}^{n}$, which implies that 


$$
\begin{aligned}
\left\|\left[\Delta, \widehat{g}_{j}^{n}\right] h\right\|_{\hat{Y}_{j}^{n}}^{2} & =\int_{\hat{B}_{j}^{n}}\left(1+\left(\delta\left|x-\widehat{p}_{j}^{n}\right|\right)^{2+\alpha}\right)\left(2 \nabla \widehat{g}_{j}^{n} \cdot \nabla h+\left(\Delta \widehat{g}_{j}^{n}\right) h\right)^{2} \delta^{2} d x \\
& \leq C \int_{\hat{B}_{j}^{n}}\left(\left|\Delta \widehat{g}_{j}^{n}\right|^{2} h^{2}+\left|\nabla \widehat{g}_{j}^{n}\right|^{2}|\nabla h|^{2}\right) \delta^{2} d x \\
& \leq C \delta^{4} \int_{\hat{B}_{j}^{n}} h^{2} \delta^{2} d x+C \delta^{2} \int_{\hat{B}_{j}^{n}}|\nabla h|^{2} \delta^{2} d x .
\end{aligned}
$$

Here, we show the following.

LEMMA 12. We have

$$
\int_{\hat{B}_{j}^{n}}\left(h^{2}+|\nabla h|^{2}\right) \delta^{2} d x \leq C \sup _{k, l}\left\|\widehat{\varphi}_{l}^{k} h\right\|_{\hat{X}_{l}^{k}}^{2}
$$

for any $h \in \widehat{H}_{\delta}$.

Proof. Given $(n, j) \in \mathbf{Z}^{2} \times\{1, \ldots, s\}$, we put

$$
J_{j}^{n}=\left\{(k, l) \in \mathbf{Z}^{2} \times\{1, \ldots, s\} \mid \widehat{B}_{l}^{k} \cap \widehat{B}_{j}^{n} \neq \emptyset\right\} .
$$

The cardinality of $J_{j}^{n}$ satisfies $|J| \equiv \sup _{n, j}\left|J_{j}^{n}\right|<+\infty$, because the periodic covering $\left\{\widehat{B}_{j}^{n}\right\}_{n, j}$ of $\mathbf{R}^{2}$ is locally finite. Therefore, Schwarz' inequality guarantees for any $x \in \widehat{B}_{j}^{n}$ that

$$
1 \equiv \sum_{(k, l) \in J_{j}^{n}} \widehat{\varphi}_{l}^{k}(x) \leq\left\{\sum_{(k, l) \in J_{j}^{n}} \widehat{\varphi}_{l}^{k}(x)^{2}\right\}^{1 / 2}\left\{\sum_{(k, l) \in J_{j}^{n}} 1^{2}\right\}^{1 / 2} \leq|J| \sum_{(k, l) \in J_{j}^{n}} \widehat{\varphi}_{l}^{k}(x)^{2}
$$

and hence

$$
\begin{aligned}
\int_{\hat{B}_{j}^{n}} h^{2} \delta^{2} d x & \leq|J| \sum_{(k, l) \in J_{j}^{n}} \int_{\hat{B}_{j}^{n} \cap \hat{B}_{l}^{k}}\left(\widehat{\varphi}_{l}^{k}\right)^{2} h^{2} \delta^{2} d x \\
& \leq C \sup _{(k, l) \in J_{j}^{n}} \int_{\hat{B}_{l}^{k}} \frac{\left(\widehat{\varphi}_{l}^{k} h\right)^{2} \delta^{2}}{\left(1+\left(\delta\left|x-\widehat{p}_{l}^{k}\right|\right)^{2+\alpha}\right)} d x \leq C \sup _{k, l}\left\|\widehat{\varphi}_{l}^{k} h\right\|_{\hat{X}_{l}^{k}}^{2}
\end{aligned}
$$

because

$$
C^{-1} \leq 1+\left(\delta\left|x-\widehat{p}_{j}^{n}\right|\right)^{2+\alpha} \leq C
$$

for all $x \in \widehat{B}_{j}^{n}$. Similarly, we have

$$
\begin{aligned}
\int_{\hat{B}_{j}^{n}}|\nabla h|^{2} \delta^{2} d x & \leq|J| \sum_{(k, l) \in J_{j}^{n}} \int_{\hat{B}_{j}^{n} \cap \hat{B}_{l}^{k}}\left(\widehat{\varphi}_{l}^{k}\right)^{2}|\nabla h|^{2} \delta^{2} d x \leq C \sum_{k, l} \int_{\hat{B}_{l}^{k}}\left|\widehat{\varphi}_{l}^{k} \nabla h\right|^{2} \delta^{2} d x \\
& \leq C \sup _{k, l} \int_{\hat{B}_{l}^{k}}\left|\nabla\left(\widehat{\varphi}_{l}^{k} h\right)\right|^{2} \delta^{2} d x+C \sup _{k, l} \int_{\hat{B}_{l}^{k}}\left|\nabla \widehat{\varphi}_{l}^{k}\right|^{2} h^{2} \delta^{2} d x \\
& \leq C \sup _{k, l} \int_{\hat{B}_{l}^{k}}\left|\nabla\left(\widehat{\varphi}_{l}^{k} h\right)\right|^{2} \delta^{2} d x+C \delta^{2} \sup _{k, l} \int_{\hat{B}_{l}^{k}} h^{2} \delta^{2} d x
\end{aligned}
$$

since $\left|\widehat{\varphi}_{l}^{k} \nabla h\right| \leq\left|\nabla\left(\widehat{\varphi}_{l}^{k} h\right)\right|+\left|\nabla \widehat{\varphi}_{l}^{k}\right||h|$. For the first term of the right-hand side, we note that $\widehat{\varphi}_{l}^{k} h \in C_{c}^{1}\left(\widehat{B}_{l}^{k}\right)$ by Sobolev's imbedding theorem, and hence it follows from Young's 
inequality that

$$
\begin{aligned}
\int_{\hat{B}_{l}^{k}}\left|\nabla\left(\widehat{\varphi}_{l}^{k} h\right)\right|^{2} \delta^{2} d x \leq & \int_{\hat{B}_{l}^{k}}\left|\left(\widehat{\varphi}_{l}^{k} h\right) \Delta\left(\widehat{\varphi}_{l}^{k} h\right)\right| \delta^{2} d x \\
\leq & \frac{1}{2} \int_{\hat{B}_{l}^{k}} \frac{\left(\widehat{\varphi}_{l}^{k} h\right)^{2} \delta^{2}}{\left(1+\left(\delta\left|x-\widehat{p}_{l}^{k}\right|\right)^{2+\alpha}\right)} d x \\
& +\frac{1}{2} \int_{\hat{B}_{l}^{k}}\left|\Delta\left(\widehat{\varphi}_{l}^{k} h\right)\right|^{2}\left(1+\left(\delta\left|x-\widehat{p}_{l}^{k}\right|\right)^{2+\alpha}\right) \delta^{2} d x \\
\leq & \frac{1}{2}\left\|\widehat{\varphi}_{l}^{k} h\right\|_{\hat{X}_{l}^{k}}^{2}+\frac{1}{2}\left\|\Delta\left(\widehat{\varphi}_{l}^{k} h\right)\right\|_{\hat{Y}_{l}^{k}}^{2} \leq\left\|\widehat{\varphi}_{l}^{k} h\right\|_{\hat{X}_{l}^{k}}^{2}
\end{aligned}
$$

Therefore, combining (5.26), (5.27), and (5.25), we obtain

$$
\int_{\hat{B}_{j}^{n}}|\nabla h|^{2} \delta^{2} d x \leq C\left(1+\delta^{2}\right) \sup _{k, l}\left\|\widehat{\varphi}_{l}^{k} h\right\|_{\hat{X}_{l}^{k}}^{2}
$$

and the proof is complete.

From (5.21) and Lemma 12, we obtain

$$
\left\|\left[\Delta, \widehat{g}_{j}^{n}\right] h\right\|_{\hat{Y}_{j}^{n}} \leq C\left(\delta+\delta^{2}\right) \sup _{k, l}\left\|\widehat{\varphi}_{l}^{k} h\right\|_{\hat{X}_{l}^{k}}
$$

and thus (5.20) follows. Therefore, we have from (5.18) and (5.19) that

$$
\left\|S_{\varepsilon, \delta} A_{\varepsilon, \delta}-I_{\hat{H}_{\delta}}\right\|=\frac{C}{\varepsilon^{2}} O(\delta) \rightarrow 0
$$

as $\delta \downarrow 0$. On the other hand,

$$
A_{\varepsilon, \delta} S_{\varepsilon, \delta}=I_{\hat{Y}_{\delta}}+\sum_{n, j} \widehat{g}_{j}^{n}\left(A_{\varepsilon, \delta}-\widehat{A}_{\varepsilon, j}^{n}\right)\left(\widehat{A}_{\varepsilon, j}^{n}\right)^{-1} \widehat{g}_{j}^{n}+\sum_{n, j}\left[A_{\varepsilon, \delta}, \widehat{g}_{j}^{n}\right]\left(\widehat{A}_{\varepsilon, j}^{n}\right)^{-1} \widehat{g}_{j}^{n},
$$

and $\left\|A_{\varepsilon, \delta} S_{\varepsilon, \delta}-I_{\hat{Y}_{\delta}}\right\| \rightarrow 0$ follows similarly.

Therefore, both $S_{\varepsilon, \delta} A_{\varepsilon, \delta}: \widehat{H}_{\delta} \rightarrow \widehat{H}_{\delta}$ and $A_{\varepsilon, \delta} S_{\varepsilon, \delta}: \widehat{Y}_{\delta} \rightarrow \widehat{Y}_{\delta}$ are invertible with $\left\|\left(A_{\varepsilon, \delta} S_{\varepsilon, \delta}\right)^{-1}\right\| \leq 2$ and $\left\|\left(S_{\varepsilon, \delta} A_{\varepsilon, \delta}\right)^{-1}\right\| \leq 2$ uniformly for $0<\delta \ll \varepsilon \ll 1$. Thus, $\left(A_{\varepsilon, \delta}\right)^{-1}$ : $\widehat{Y}_{\delta} \rightarrow \widehat{H}_{\delta}$ exists as

$$
\left(A_{\varepsilon, \delta}\right)^{-1}=S_{\varepsilon, \delta}\left(A_{\varepsilon, \delta} S_{\varepsilon, \delta}\right)^{-1}=\left(S_{\varepsilon, \delta} A_{\varepsilon, \delta}\right)^{-1} S_{\varepsilon, \delta}
$$

We also have by (5.15) that

$$
\left\|\left(A_{\varepsilon, \delta}\right)^{-1} h\right\|_{\hat{H}_{\delta}}=\left\|\left(S_{\varepsilon, \delta} A_{\varepsilon, \delta}\right)^{-1} S_{\varepsilon, \delta} h\right\|_{\hat{H}_{\delta}} \leq 2\left\|S_{\varepsilon, \delta} h\right\|_{\hat{H}_{\delta}} \leq \frac{C}{\varepsilon^{2}}\|h\|_{\hat{Y}_{\delta}}
$$

for $h \in \widehat{Y}_{\delta}$. The proof of Theorem 3 is complete.

6. Completion of proof. We are in a position to apply Banach's fixed point theorem to the functional equation $F_{\varepsilon, \delta}(z)=0$. Henceforth, we put $B_{r}=\left\{z \in \widehat{H}_{\delta} \mid\|z\|_{\hat{H}_{\delta}} \leq r\right\}$ for $r>0$, and introduce the nonlinear mapping $G_{\varepsilon, \delta}: B_{1} \rightarrow \widehat{H}_{\delta}$ by

$$
G_{\varepsilon, \delta}(z)=z-\left(A_{\varepsilon, \delta}\right)^{-1} F_{\varepsilon, \delta}(z) .
$$

Thus, we are seeking a fixed point of $G_{\varepsilon, \delta}$ in $B_{1}$. Actually, this is done by the following. (See Theorem 7.1 of [9].) 
THEOREM 4. For any $0<\varepsilon \ll 1$ there exists $\delta_{\varepsilon}>0$ satisfying $\lim _{\varepsilon \downarrow 0} \delta_{\varepsilon}=0$ such that if $\delta \in\left(0, \delta_{\varepsilon}\right)$ there is a unique fixed point $z_{\varepsilon, \delta}^{*}$ in $B_{R_{\delta}}$ of $G_{\varepsilon, \delta}$, where $R_{\delta}=\delta^{\beta}$ with $\beta \in(1,2)$. Proof. We show that $G_{\varepsilon, \delta}$ is a contractive self-map on $B_{r}$ for $r=R_{\delta}=O\left(\delta^{\beta}\right)$ chosen below. In fact, we have

$$
D G_{\varepsilon, \delta}(z)=I-A_{\varepsilon, \delta}^{-1} D F_{\varepsilon, \delta}(z)=A_{\varepsilon, \delta}^{-1}\left(A_{\varepsilon, \delta}-D F_{\varepsilon, \delta}(z)\right)
$$

and hence Theorem 3 guarantees that

$$
\left\|D G_{\varepsilon, \delta}(z)\right\| \leq\left\|A_{\varepsilon, \delta}^{-1}\right\|\left\|D F_{\varepsilon, \delta}(z)-A_{\varepsilon, \delta}\right\| \leq \frac{C}{\varepsilon^{2}}\left\|D F_{\varepsilon, \delta}(z)-A_{\varepsilon, \delta}\right\|
$$

for any $z \in B_{1}$.

Now, we have

$$
\begin{aligned}
D F_{\varepsilon, \delta}(z)= & \Delta+\sum_{n, j} \widehat{\varphi}_{j}^{n} \widehat{\rho}_{j}^{n} e^{\varepsilon^{2}\left(\hat{w}_{j}^{n}+\hat{v}_{\varepsilon, j}^{n}\right)} e^{\varepsilon^{2} z} R_{j}^{n}(x) \\
& -2 \varepsilon^{2} \sum_{n, j} \widehat{\varphi}_{j}^{n}\left(\widehat{\rho}_{j}^{n}\right)^{2} e^{2 \varepsilon^{2}\left(\hat{w}_{j}^{n}+\hat{v}_{\varepsilon, j}^{n}\right)} e^{2 \varepsilon^{2} z} R_{j}^{n}(x)^{2}
\end{aligned}
$$

and hence

$$
\begin{aligned}
\|\left(D F_{\varepsilon, \delta}(z)-\right. & \left.A_{\varepsilon, \delta}\right) h\left\|_{\hat{Y}_{\delta}}=\right\|\left(D F_{\varepsilon, \delta}(z)-D F_{\varepsilon, \delta}(0)\right) h \|_{\hat{Y}_{\delta}} \\
= & \sup _{n, j}\left\|\widehat{\varphi}_{j}^{n}\left(D F_{\varepsilon, \delta}(z)-D F_{\varepsilon, \delta}(0)\right) h\right\|_{\hat{Y}_{j}^{n}} \\
\leq & C \sup _{n, j}\left\|\widehat{\varphi}_{j}^{n} \widehat{\rho}_{j}^{n} e^{\varepsilon^{2}\left(\hat{w}_{j}^{n}+\hat{v}_{\varepsilon, j}^{n}\right)} R_{j}^{n}(x)\left(e^{\varepsilon^{2} z}-1\right) \widehat{\varphi}_{j}^{n} h\right\|_{\hat{Y}_{j}^{n}} \\
& +C \varepsilon^{2} \sup _{n, j}\left\|\widehat{\varphi}_{j}^{n}\left(\widehat{\rho}_{j}^{n}\right)^{2} e^{2 \varepsilon^{2}\left(\hat{w}_{j}^{n}+\hat{v}_{\varepsilon, j}^{n}\right)} R_{j}^{n}(x)^{2}\left(e^{2 \varepsilon^{2} z}-1\right) \widehat{\varphi}_{j}^{n} h\right\|_{\hat{Y}_{j}^{n}}
\end{aligned}
$$

for any $h \in \widehat{H}_{\delta}$. Because of the maximum principle to (4.14) we have $\widehat{u}_{j}^{n} \leq 0$ and hence it follows from (4.19) that

$$
\varepsilon^{2} \widehat{\rho}_{j}^{n} e^{\varepsilon^{2}\left(\hat{w}_{j}^{n}+\hat{v}_{\varepsilon, j}^{n}\right)} R_{j}^{n} \leq 1
$$

in $\widehat{B}_{j}^{n}$. We also have

$$
\left|e^{\varepsilon^{2} z(x)}-1\right|=\left|\frac{d}{d t} \int_{0}^{1} e^{\varepsilon^{2} t z(x)} d t\right| \leq \varepsilon^{2}\|z\|_{\infty} e^{\varepsilon^{2}\|z\|_{\infty}}
$$

for all $x \in \mathbf{R}^{2}$, and Sobolev's inequality implies that

$$
\|u\|_{\infty} \leq C\|u\|_{W^{2,2}} \approx \frac{C}{\delta}\|u\|_{\hat{X}_{j}^{n}}
$$

for $u \in \widehat{X}_{j}^{n}$ in supp $u \subset \widehat{B}_{j}^{n}$. From those relations we obtain

$$
\begin{aligned}
& \left\|\left(D F_{\varepsilon, \delta}(z)-A_{\varepsilon, \delta}\right) h\right\|_{\hat{Y}_{\delta}} \leq C \sup _{n, j}\left\|\widehat{\varphi}_{j}^{n} z\right\|_{\infty}\left(e^{\varepsilon^{2}\|z\|_{\infty}}+e^{2 \varepsilon^{2}\|z\|_{\infty}}\right)\left\|\widehat{\varphi}_{j}^{n} h\right\|_{\hat{X}_{j}^{n}} \\
& \leq \frac{C}{\delta} \sup _{n, j}\left\|\widehat{\varphi}_{j}^{n} z\right\|_{\hat{X}_{j}^{n}}\left(2+3 \varepsilon^{2}\|z\|_{\infty}\right)\left\|\widehat{\varphi}_{j}^{n} h\right\|_{\hat{X}_{j}^{n}} \leq \frac{C}{\delta}\|z\|_{\hat{H}_{\delta}}\left(1+\frac{C}{\delta}\|z\|_{\hat{H}_{\delta}}\right)\|h\|_{\hat{H}_{\delta}}
\end{aligned}
$$

since $e^{x}\left(1+e^{x}\right)=2+3 x+o(x)$ as $x \rightarrow 0$.

We conclude from (6.6) and (6.2) that

$$
\left\|D G_{\varepsilon, \delta}(z)\right\| \leq \frac{1}{2} \quad\left(z \in B_{R_{\delta}}\right)
$$


for $0<\varepsilon \ll 1$ if

$$
\frac{C R_{\delta}}{\delta}\left(1+\frac{C}{\delta} R_{\delta}\right) \leq \frac{1}{2} \varepsilon^{2}
$$

If we take $R_{\delta}=\delta^{\beta}$ for $\beta \in(1,2)$, this requirement (6.8) follows from

$$
C \delta^{\beta-1}\left(1+C \delta^{\beta-1}\right) \leq \frac{\varepsilon^{2}}{2}
$$

or equivalently, $\delta^{\beta-1} \leq \frac{\varepsilon^{2}}{4 C}$. Therefore, setting

$$
\delta_{\varepsilon}=\left(\frac{\varepsilon^{2}}{4 C}\right)^{1 /(\beta-1)}
$$

we see that (6.7) holds for $\delta \in\left(0, \delta_{\varepsilon}\right)$ and $R_{\delta}=\delta^{\beta}$, which implies that $G_{\varepsilon, \delta}$ is a contraction on $B_{R_{\delta}}$.

Next, we have for $z \in B_{R_{\delta}}$ that

$$
\begin{aligned}
\left\|G_{\varepsilon, \delta}(z)\right\|_{\hat{H}_{\delta}} & \leq\left\|G_{\varepsilon, \delta}(z)-G_{\varepsilon, \delta}(0)\right\|_{\hat{H}_{\delta}}+\left\|G_{\varepsilon, \delta}(0)\right\|_{\hat{H}_{\delta}} \\
& \leq \frac{1}{2}\|z\|_{\hat{H}_{\delta}}+\left\|A_{\varepsilon, \delta}^{-1} F_{\varepsilon, \delta}(0)\right\|_{\hat{H}_{\delta}}
\end{aligned}
$$

and it follows from Theorem 3 and Lemma 10 that

$$
\left\|A_{\varepsilon, \delta}^{-1} F_{\varepsilon, \delta}(0)\right\|_{\hat{H}_{\delta}} \leq\left\|A_{\varepsilon, \delta}^{-1}\right\|\left\|F_{\varepsilon, \delta}(0)\right\|_{\hat{Y}_{\delta}} \leq \frac{C}{\varepsilon^{4}} \delta^{2}|\log \delta| .
$$

Therefore, if $\frac{C}{\varepsilon^{4}} \delta^{2}|\log \delta| \leq \frac{1}{2} R_{\delta}$ then $G_{\varepsilon, \delta}\left(B_{R_{\delta}}\right) \subset B_{R_{\delta}}$. Because $R_{\delta}=\delta^{\beta}$, this is equivalent to $\delta^{2-\beta}|\log \delta| \leq \frac{1}{2 C} \varepsilon^{4}$, which means that $\delta \in\left(0, \delta_{\varepsilon}\right)$ for some $\delta_{\varepsilon}>0$ with $\lim _{\varepsilon \downarrow 0} \delta_{\varepsilon}=0$. The proof is complete.

Theorem 4 assures that

$$
\widehat{u}_{\varepsilon, \delta}(x)=\sum_{n \in \mathbf{Z}^{2}} \sum_{j=1}^{s} \widehat{\varphi}_{j}^{n}(x)\left(\log \left(\varepsilon^{2} \widehat{\rho}_{j}^{n}(x)\right)+\varepsilon^{2} \widehat{w}_{j}^{n}(x)+\varepsilon^{2} \widehat{v}_{\varepsilon, j}^{n}(x)\right)+\varepsilon^{2} z_{\varepsilon, \delta}^{*}(x)
$$

is a solution to $(2.2)$. On the other hand, it is obvious that $z_{\varepsilon, \delta}^{*}\left(\cdot+\widehat{e}_{k}\right) \in B_{R_{\delta}}$ and $F_{\varepsilon, \delta}\left(z_{\varepsilon, \delta}^{*}\left(\cdot+\widehat{e}_{k}\right)\right)=F_{\varepsilon, \delta}\left(z_{\varepsilon, \delta}^{*}\right)=0$. Hence from the uniqueness of the fixed point of $G_{\varepsilon, \delta}$ in $B_{R_{\delta}}$, we have $z_{\varepsilon, \delta}^{*}\left(x+\widehat{e}_{k}\right)=z_{\varepsilon, \delta}^{*}(x)$ for any $x \in \mathbf{R}^{2}$ and $k=1,2$. This implies $\widehat{u}_{\varepsilon, \delta}\left(x+\widehat{e}_{k}\right)=\widehat{u}_{\varepsilon, \delta}(x)$ for $k=1,2$ and $\widehat{u}_{\varepsilon, \delta}$ is a doubly periodic solution to (2.2).

Back to (1.1), given $\kappa=2 \varepsilon \delta>0$ with $0<\varepsilon \ll 1$, we have $\delta_{\varepsilon}>0$ such that if $\delta \in\left(0, \delta_{\varepsilon}\right)$ there is a solution $u_{\varepsilon, \delta}$ to (1.1). It has the form

$$
u_{\varepsilon, \delta}(x)=\sum_{j=1}^{s} \phi_{j}(x) u_{\varepsilon, m_{j}}^{*}\left(\left|x-p_{j}\right| / \delta\right)+\varepsilon^{2} z_{\varepsilon, \delta}^{*}(x / \delta)
$$

for $x \in \Omega$, with $u_{\varepsilon, m_{j}}^{*}\left(\left|\cdot-p_{j}\right| / \delta\right)$ standing for the radially symmetric single vortex solution with the vortex point $p_{j}$ and multiplicity $m_{j}$. Once this expression is obtained, we can evaluate the asymptotic behavior of $\left|\phi_{\kappa}\right|=\exp \left(u_{\varepsilon, \delta} / 2\right)$ exactly in the same way as in Proposition 8 of [9]. We just state it for completeness.

TheOREM 5. If $\kappa=2 \varepsilon \delta$ with $0<\varepsilon \ll 1$ and $\delta \in\left(0, \delta_{\varepsilon}\right)$, then $\left|\phi_{\kappa}\right|=\exp \left(u_{\varepsilon, \delta} / 2\right)$ defined for $u_{\varepsilon, \delta}$ given by (6.11) satisfies the following:

(1) $\left|\phi_{\kappa}\right|<1$ on $\Omega$ and vanishes exactly at the vortex points $p_{j}$. 
(2) $\left|\phi_{\kappa}\right| \rightarrow 0$ in $C_{l o c}^{q}\left(\Omega \backslash\left\{p_{1}, \ldots, p_{s}\right\}\right)$ for any $q \geq 0$ as $\kappa \rightarrow 0$.

(3) $\frac{4}{\kappa^{2}}\left|\phi_{\kappa}\right|^{2}\left(1-\left|\phi_{\kappa}\right|^{2}\right) \rightarrow 4 \pi \sum_{j=1}^{s} m_{j} \delta_{p_{j}}$ in the sense of measures on $\Omega$ as $\kappa \downarrow 0$.

Note. After completing the work, we noticed that Nolasco's paper has appeared in Comm. Pure Appl. Math. 56 (2003), 1752-1780. In that paper, she provided several modifications of the original preprint ([9]), and the numbers of formulas and propositions are changed. In particular, formula (6.34), Theorem 7.1, and Proposition 8 referred to in our paper are formula (6.36), Theorem 6.6, and Proposition 7.1 of the modified version, respectively. Lemma 6.4 of the original paper is corrected just as we pointed out (Lemma 11), but other parts still seem to need modifications. So far, we have confirmed that her result is correct in the case of $s \geq 2$, but fortunately, this is also the case for $s=1$. It is proven by a slightly different covering of vortices, and detailed arguments will be published elsewhere.

\section{References}

[1] L. Caffarelli and Y. Yang, Vortex condensation in the Chern-Simons-Higgs model: an existence theorem, Comm. Math. Phys. 168 (1995), 321-366.

[2] D. Chae and O. Imanuvilov, The existence of non-topological multivortex solutions in the relativistic selfdual Chern-Simons theory, Comm. Math. Phys. 215 (2000), 119-142.

[3] W. Ding, J. Jost, J. Li, X. Peng and G. Wang, Self duality equations for Ginzburg-Landau and Seiberg-Witten type functional with $6^{\text {th }}$ order potential, Comm. Math. Phys. 217 (2001), 383-407.

[4] W. Ding, J. Jost, J. Li and G. Wang, An analysis of the two-vortex case in the ChernSimons-Higgs model, Calc. Var. and PDE 7 (1998) 87-97.

[5] G. Dunne, Self-Dual Chern-Simons Theories, Springer, Berlin, 1995.

[6] I. C. Gohberg and M. G. Krein, The basic propositions on defect numbers, root numbers and indices of linear operators, Uspehi Mat. Nauk 12 (1957), 43-118 (in Russian); English transl.: Amer. Math. Soc. Translations Ser. 213 (1960), 185-264.

[7] M. Nolasco and G. Tarantello, On a sharp Sobolev-type inequality on two-dimensional compact manifolds, Arch. Rational Mech. Anal. 145 (1998), 161-195.

[8] M. Nolasco and G. Tarantello, Double vortex condensates in the Chern-Simons-Higgs theory, Calc. Var. and PDE 9 (1999), 31-94.

[9] M. Nolasco, Non-topological N-vortex condensates for the self-dual Chern-Simons theory, preprint.

[10] G. Tarantello, Multiple condensate solutions for the Chern-Simons-Higgs theory, J. Math. Phys. 37 (1996), 3769-3796.

[11] Y. Yang, Solitons in Field Theory and Nonlinear Analysis, Springer, New York, 2001. 